Draft VERSION July 14, 2021

Preprint typeset using $\mathrm{LAT}_{\mathrm{E}} \mathrm{X}$ style AASTeX6 v. 1.0

\title{
THE STATISTICS OF RADIO ASTRONOMICAL POLARIMETRY: DISJOINT, SUPERPOSED, AND COMPOSITE SAMPLES
}

\author{
W. VAN STRATEN \\ Centre for Astrophysics and Supercomputing, Swinburne University of Technology, Hawthorn, VIC 3122, Australia \\ ARC Centre of Excellence for All-sky Astrophysics (CAASTRO) \\ and
}

Institute for Radio Astronomy \& Space Research, Auckland University of Technology, Private Bag 92006, Auckland 1142, New Zealand

\author{
C. Tiburzi \\ Max-Planck-Institut für Radioastronomie, Auf dem Hügel 69, 53121 Bonn, Germany \\ and \\ Universität Bielefeld, Fakultät für Physik, Universitätsstr. 25, D-33615 Bielefeld, Germany
}

(Received)

\begin{abstract}
A statistical framework is presented for the study of the orthogonally polarized modes of radio pulsar emission via the covariances between the Stokes parameters. To accommodate the typically heavytailed distributions of single-pulse radio flux density, the fourth-order joint cumulants of the electric field are used to describe the superposition of modes with arbitrary probability distributions. The framework is used to consider the distinction between superposed and disjoint modes with particular attention to the effects of integration over finite samples. If the interval over which the polarization state is estimated is longer than the timescale for switching between two or more disjoint modes of emission, then the modes are unresolved by the instrument. The resulting composite sample mean exhibits properties that have been attributed to mode superposition, such as depolarization. Because the distinction between disjoint modes and a composite sample of unresolved disjoint modes depends on the temporal resolution of the observing instrumentation, the arguments in favour of superposed modes of pulsar emission are revisited and observational evidence for disjoint modes is described. In principle, the four-dimensional covariance matrix that describes the distribution of sample mean Stokes parameters can be used to distinguish between disjoint modes, superposed modes, and a composite sample of unresolved disjoint modes. More comprehensive and conclusive interpretation of the covariance matrix requires more detailed consideration of various relevant phenomena, including temporally correlated subpulse modulation (e.g. jitter), statistical dependence between modes (e.g. covariant intensities and partial coherence), and multipath propagation effects (e.g. scintillation and scattering). Unpublished supplementary material is appended after the bibliography.
\end{abstract}

Keywords: methods: data analysis — methods: statistical — polarization — pulsars: general — techniques: polarimetric

\section{INTRODUCTION}

The higher-order statistics of electromagnetic radiation have long been used to discover and study radio pulsars. The fluctuation power spectrum is employed to detect periodic signals in pulsar survey data

willem.van.straten@aut.ac.nz (e.g. Burns \& Clark 1969; Ransom et al. 2002); the longitude-resolved modulation index is used to study the radio pulsar emission mechanism (e.g. Taylor et al. 1975; Krishnamohan \& Downs 1983; Jenet \& Gil 2003; Weltevrede et al. 2007); correlated and periodic structure in the variability of pulsar signals, such as drifting subpulses, are detected and studied using longitude resolved and two-dimensional fluctuation spectra (e.g. Backer 1970; Edwards \& Stappers 2002); and the sec- 
ondary dynamic spectrum reveals information about the turbulent structure of the ionized interstellar medium along the pulsar line of sight (e.g. Stinebring et al. 2001; Walker et al. 2004; Cordes et al. 2006). All of the above quantities involve fourth-order moments of the electric field that characterize variability in the flux density of the pulsar signal.

The fourth moments of the electric field have also been used to study variability in the polarization of pulsar radiation. In many pulsars, over limited ranges of pulsar longitude, the emission is observed to switch between one of two orthogonally polarized states, or modes (e.g. Ekers \& Moffet 1969; Taylor et al. 1971; Manchester et al. 1975; Backer \& Rankin 1980; Stinebring et al. 1984). The degree of polarization of the modes and the time scales for mode switching (e.g. microstructure and subpulse polarization fluctuations) have been inferred from the autocorrelation functions of the Stokes parameters (e.g. Cordes 1976; Cordes \& Hankins 1977; Cordes et al. 2004). In several cases, the observed modes are not perfectly orthogonally polarized (e.g. Backer \& Rankin 1980; Stinebring et al. 1984; Gil et al. 1991; McKinnon 2003a) and surprising annular distributions of single-pulse polarization states on the surface of the Poincaré sphere have also been observed, which may be indicative of stochastic generalized Faraday rotation in the pulsar magnetosphere (Edwards \& Stappers 2004).

Cordes et al. (1978), hereafter CRB, first proposed using the (non-central) second moments of the linearly polarized and total intensities to determine if the modes are disjoint, such that only one mode is observed at a given instant, or if the signal is an incoherent superposition of the two modes. CRB also argued that the high degree of correlation between the handedness of circular polarization and the position angle of the linearly polarized flux is further evidence of the mutual exclusivity of the modes. However, this correlation is more simply interpreted as evidence for the ellipticity of the modes (e.g. Harding \& Tademaru 1981; Allen \& Melrose 1982; Karastergiou et al. 2003).

Over the decades following CRB, several studies considered the distinction between disjoint and superposed modes (e.g. Stinebring et al. 1984; McKinnon \& Stinebring 1998), and a wide variety of statistical approaches to the study of orthogonally polarized modes have been developed (e.g. McKinnon \& Stinebring 2000; McKinnon 2002, 2003b, 2006). Edwards \& Stappers (2004) and McKinnon (2004) independently introduced techniques based on principal component analysis of the $3 \times 3$ covariance matrix of the Stokes polarization vector with the respective goals of characterizing the non-orthogonality of the modes and testing the hypothesis that the excess broadening of position angle histograms (first noted by Stinebring et al. 1984) is due to additional randomly polarized radiation. Influenced by these studies, van Straten (2009) analyzed the $4 \times 4$ covariance matrix of the Stokes parameters and demonstrated that additional randomly polarized radiation is not required. Rather, the observed variance of the polarized flux is consistent with the self noise intrinsic to the bright pulsars on which single-pulse studies typically focus.

The statistical analysis presented by van Straten (2009) is valid only when the components of the electric field vector are normally distributed. However, pulsars typically exhibit heavy-tailed (e.g. power-law and lognormal) distributions of longitude-resolved single-pulse flux density (e.g. Cairns et al. 2003; Osłowski et al. 2014). Therefore, one of the main aims of this paper is to extend (and correct) the statistical framework of van Straten (2009) so that it can be applied to nonnormal distributions.

As in van Straten (2009), the framework presented in this paper differentiates between the statistics of the $i n$ stantaneous Stokes parameters, which are derived from a single instance of the electric field, and the sample mean Stokes parameters, which are computed by averaging over a finite sample of instances of the electric field. The instantaneous Stokes parameters, also known as the unaveraged or unsmoothed Stokes parameters, are typically encountered only when studying the autocorrelation functions of the Stokes parameters as a function of lag (e.g. Cordes 1976; Cordes \& Hankins 1977; Cordes et al. 2004) or when presenting the polarization of giant pulses at the highest available time resolution (e.g. Heiles et al. 1970; Cognard et al. 1996; Hankins et al. 2003). ${ }^{1}$ The sample mean Stokes parameters are more commonly encountered in the study of single pulses from radio pulsars, where the finite samples are defined by the evenly spaced intervals of pulsar longitude, also known as phase bins, into which the signal is divided.

The distinction between instantaneous and sample mean statistics reaffirms the fundamental importance of instrumental resolution when studying orthogonally polarized modes. Whereas instantaneous Stokes parameters admit only disjoint or superposed modes, the sample mean Stokes parameters may include statistical samples that are composed of a union of sub-samples drawn from mutually exclusive populations. In other words, after integration, disjoint modes may be unresolved by

\footnotetext{
${ }^{1}$ See van Straten (2009) for a detailed discussion of the fundamental limitations and pitfalls associated with studying giant pulse polarization via the instantaneous Stokes parameters, which on their own have no statistically significant physical meaning.
} 
the instrument.

Observational evidence of disjoint modes is presented by Cordes \& Hankins (1977), who find that micropulse structures on the shortest time scales are more polarized than the longer time scale subpulse structures in which they are embedded. They also observe that transitions between orthogonally polarized modes preferentially occur on the edges of micropulses and conclude that the variability of micropulse polarization depolarizes the signal when it is smoothed on a time scale greater than the characteristic width of the micropulse structures. That is, the signal is depolarized when the disjoint modes are unresolved. As discussed in more detail in Appendix A, similar evidence of unresolved disjoint modes is presented by Gangadhara et al. (1999), who demonstrate that the degree of polarization of single-pulse observations is higher when the integration interval is shorter; that is, at higher time resolution, the disjoint modes are better resolved.

Integration over a sample that is composed of mutually exclusive and orthogonally polarized sub-samples depolarizes the resulting sample mean Stokes parameters. This provides an alternative explanation for depolarization, which is more commonly interpreted as evidence of mode superposition (e.g. Stinebring et al. 1984; McKinnon \& Stinebring 1998; Karastergiou et al. 2011). Because the degree of polarization, and therefore the previously proposed distinction between disjoint and superposed modes, depends on the temporal resolution of the instrument used to record the experimental data, the observational evidence that has been presented in support of superposed modes should be revisited.

Toward this end, and motivated by the additional insights that can be gained through analysis of the fourdimensional fourth-order moments of the electric field, the primary aim of this paper is to further develop a statistical framework for the study of orthogonally polarized modes via the covariances between the Stokes parameters. To date, studies of variability in the polarized emission from pulsars have focused on sources bright enough to be detected on every rotation. In contrast, the approach described in this paper does not require detection of individual pulses and can be applied to average pulse profiles of arbitrary integration length. Therefore, the proposed framework can be employed to study sources that are either insufficiently bright to be clearly detected in single-pulse data or for which recording and offline analysis of such data is prohibitively expensive, which are some of the reasons why single-pulse studies of millisecond pulsar polarization are relatively rare and recent (e.g. Osłowski et al. 2014; Liu et al. 2015). Experiments based on statistical interpretation of moments should be less influenced by the idiosyncrasies of the relatively few brightest (and more slowly spinning) sources and thereby have the potential to enable more statistically significant conclusions about the entire pulsar population.

Following a review of fourth-order statistics in Section 1.1, some relevant linear algebra and the covariances between the instantaneous Stokes parameters are presented in Section 2. In Section 3, the covariances between the sample mean Stokes parameters are derived for the following three distinct and idealized combinations of statistical samples.

1. Disjoint samples: Every instance of the electric field vector in a given sample is exclusively from only one of the two modes of emission; i.e. the disjoint modes are resolved by the instrument.

2. Superposed samples: Each instance of the electric field vector is an incoherent sum of the electric fields from the two modes.

3. Composite samples: Each sample is a union of sub-samples of instances of the electric field vector from each of the two modes of emission; i.e. the disjoint modes are unresolved by the instrument.

In Section 4, the results of this analysis are interpreted with attention to the impact of various physical phenomena, including amplitude modulation, partial mode coherence, interstellar scintillation, and superposition of signal and noise. The mathematical equations presented throughout this paper are verified using a Monte Carlo simulation that is described in the Appendix and the results of this study are summarized and discussed in Section 5 .

\subsection{Review of Fourth-Order Statistics}

This section reviews definitions and concepts that are relevant to the instantaneous Stokes parameters, beginning with the fourth-order statistics of complex-valued random scalar variables. One of the central mathematical results of this paper is derived using the cumulants of a probability distribution, which are closely related to its moments (e.g. Kendall et al. 1987). As demonstrated in the following sections, the cumulants have an important property that is not shared by the moments of a distribution. For any two statistically independent random variables, $x$ and $y$, the cumulants of the sum $z=x+y$ are equal to the sums of the cumulants of $x$ and $y$. The moments and cumulants of a distribution are equal only up to third order and, to highlight the fundamental differences between them, the following section begins with a simple demonstration that the fourth moment of a sum of two random variables is not equal to the sum of their fourth moments. A definition of the cumulants is then presented using a derivation 
that explains the origin of their additive property, which is later exploited to study the fourth-order statistics of superposed electromagnetic waves.

\subsubsection{Complex-valued Random Scalars}

The following example illustrates the utility of the higher order cumulants of a distribution when studying the superposition of electromagnetic waves. Consider a complex-valued analytic signal $z$ associated with a realvalued random variable (e.g. Bracewell 1986). Such a signal is an example of a complex circular scalar field, defined as having statistically independent and identically distributed real and imaginary components, $\operatorname{Re}[z]$ and $\operatorname{Im}[z]$ (e.g. Picinbono 1994). If $\operatorname{Re}[z]$ and $\operatorname{Im}[z]$ are also normally distributed, then the instantaneous intensity of this scalar field, $\xi=z^{*} z$, where $z^{*}$ is the complex conjugate of $z$, is distributed as $\chi^{2}$ with two degrees of freedom (the exponential distribution). Therefore the standard deviation of $\xi$ is equal to its mean; i.e. $\varsigma_{\xi}=\langle\xi\rangle$, where the angular brackets denote the expectation value, or population mean. (When averaged over a sufficiently large number of instances, the population mean and expectation value are equal.)

If $z$ is an incoherent sum of two statistically independent and normally-distributed complex circular scalar fields, i.e. $z=z_{A}+z_{B}$, then $\langle\xi\rangle=\left\langle\xi_{A}\right\rangle+\left\langle\xi_{B}\right\rangle$, where $\xi_{A}=z_{A}^{*} z_{A}, \xi_{B}=z_{B}^{*} z_{B}$, and

$$
\varsigma_{\xi}^{2}=\langle\xi\rangle^{2}=\left(\left\langle\xi_{A}\right\rangle+\left\langle\xi_{B}\right\rangle\right)^{2}=\varsigma_{A}^{2}+\varsigma_{B}^{2}+2 \varsigma_{A} \varsigma_{B},
$$

where $\varsigma_{A}=\left\langle\xi_{A}\right\rangle$ and $\varsigma_{B}=\left\langle\xi_{B}\right\rangle$ are the standard deviations of the exponentially distributed intensities, $\xi_{A}$ and $\xi_{B}$ respectively. This elementary result demonstrates the fact that, when two statistically independent fields are superposed, the variance of the intensity of the resulting sum is not equal to the sum of the variances of the intensities of the two fields. The variance of the intensity $\varsigma_{\xi}^{2}=\left\langle\xi^{2}\right\rangle-\langle\xi\rangle^{2}$, where the second moment $\left\langle\xi^{2}\right\rangle$ is equal to the fourth-order moment of $z$; therefore, this example also demonstrates that the fourth-order moment of a sum of two random variables is not equal to the sum of their fourth moments.

To derive a general expression for the variance of the instantaneous intensity of superposed fields that are not necessarily normally distributed, it is useful to exploit the relationships between the higher-order moments and cumulants of random variables. Following Amblard et al. (1996) and Eriksson et al. (2010), the characteristic function of a complex-valued circular random variable $z$ is defined as

$$
\begin{aligned}
\Phi_{z}(Z) & \equiv\left\langle\exp \left(i \operatorname{Re}\left[z^{*} Z\right]\right)\right\rangle \\
& \equiv \int e^{i \operatorname{Re}\left[z^{*} Z\right]} p_{z}(z) d z
\end{aligned}
$$

where $i=\sqrt{-1}, p_{z}(z)$ is the probability density func- tion of $z$, and $Z$ is the Fourier conjugate of $z$. That is, the above integral is equivalent to the two-dimensional Fourier transform of $p_{z}(z)$ along the real and imaginary components of $z$, for which the Fourier conjugate variables are the real and imaginary parts of $Z$, respectively. The $n^{\text {th }}$-order moment of $z$

$$
\mu_{r ; s}(z) \equiv\left\langle z^{r} z^{* s}\right\rangle=\left.\left(\frac{2}{i}\right)^{r+s} \frac{\partial^{r+s} \Phi_{z}}{\partial Z^{s} \partial Z^{* r}}\right|_{Z=0},
$$

where $r$ and $s$ are positive integers such that $r+s=n$; the above equation identifies the moments as the coefficients in a power series expansion of the characteristic function. For a given order $n$, there are $n+1$ different moments of a complex-valued random variable; however, for a circular complex variate, the only non-zero moments are those for which $r=s$.

If $z=z_{A}+z_{B}$ is the sum of two statistically independent circular complex variates $z_{A}$ and $z_{B}$, then the probability density function of $\mathrm{z}$ is the convolution of the probability density functions of $z_{A}$ and $z_{B}$; i.e.

$$
p_{z}(z)=p_{A}(z) * p_{B}(z)
$$

Furthermore, as the Fourier transform of the probability density function, the characteristic function of $z$ is the product of the characteristic functions of $z_{A}$ and $z_{B}$; i.e.

$$
\Phi_{z}(Z)=\Phi_{A}(Z) \Phi_{B}(Z) .
$$

Finally, the natural logarithm of the characteristic function of $z$, known as the secondary characteristic function,

$$
\Psi_{z}(Z) \equiv \log \left[\Phi_{Z}(Z)\right]
$$

is simply the sum of the secondary characteristic functions of $z_{A}$ and $z_{B}$; i.e.

$$
\Psi_{z}(Z)=\Psi_{A}(Z)+\Psi_{B}(Z) .
$$

This important property is passed on to the cumulants, which are the coefficients in a power series expansion of the secondary characteristic function; i.e.

$$
\left.\kappa_{r ; s}(z) \equiv\left(\frac{2}{i}\right)^{r+s} \frac{\partial^{r+s} \Psi_{z}}{\partial Z^{s} \partial Z^{* r}}\right|_{Z=0}
$$

Therefore, $\kappa_{r ; s}(z)=\kappa_{r ; s}\left(z_{A}\right)+\kappa_{r ; s}\left(z_{B}\right)$. Using the relationship between the fourth-order cumulants and moments of circular complex variables (e.g. Mendel 1991; Eriksson et al. 2010),

$$
\kappa_{2 ; 2}(z)=\mu_{2 ; 2}(z)-2\left[\mu_{1 ; 1}(z)\right]^{2}=\varsigma_{\xi}^{2}-\langle\xi\rangle^{2},
$$

it is trivial to show that

$$
\varsigma_{\xi}^{2}=\varsigma_{A}^{2}+\varsigma_{B}^{2}+2\left\langle\xi_{A}\right\rangle\left\langle\xi_{B}\right\rangle
$$

This general expression for the variance of the instantaneous intensity of a scalar field holds regardless of the distribution of the circular complex variables $z_{A}$ and $z_{B}$ 
and, in the special case of normally distributed variates, it is consistent with Equation (1). Noting that the variance of a random variable is related to its autocorrelation function at zero lag, the above equation is consistent with Equation 16 of Rickett (1975). ${ }^{2}$

\subsubsection{Complex-valued Random Vectors}

To study the statistics of the electric field vector requires multivariate analysis and the most elegant mathematical descriptions of random vectors employ tensor algebra (e.g. McCullagh 1987; Amblard et al. 1996; Smirnov 2011). This is especially apparent when studying higher-order moments, as alternative approaches to multivariate analysis typically result in complicated expressions that involve the Kronecker product, the vectorization operator (which converts $p \times q$ matrices into $p q$-dimensional vectors), and commutation (also known as permutation) matrices; e.g. see Equation (50) of Mendel (1991), Equation (2.17) of Sultan \& Tracy (1996), and Equation (2.1.53) of Kollo \& von Rosen (2005).

Following Amblard et al. (1996), the $n^{\text {th }}$-order moments of the two-dimensional complex-valued transverse electric field vector $\boldsymbol{e}$ are given by $n+1$ rank $n$ tensors defined by

$$
\boldsymbol{\mu}_{p ; q}(\boldsymbol{e}) \equiv\left\langle\boldsymbol{e}^{\otimes p} \otimes \boldsymbol{e}^{\dagger \otimes q}\right\rangle,
$$

where $p$ and $q$ are positive integers, $p+q=n$, $e^{\dagger}$ is the Hermitian transpose of $\boldsymbol{e}, \otimes$ indicates the tensor product, and $\boldsymbol{e}^{\otimes p}$ is the $p^{\text {th }}$ tensor power of $\boldsymbol{e}$, which indicates that $e$ enters into the tensor product $p$ times. (Defined recursively, $\boldsymbol{e}^{\otimes p+1}=\boldsymbol{e}^{\otimes p} \otimes \boldsymbol{e}$.) As in the previous section, the $n^{\text {th }}$-order tensor moments are related to the characteristic function

$$
\Phi_{\boldsymbol{e}}(\boldsymbol{E}) \equiv\left\langle\exp \left(i \operatorname{Re}\left[\boldsymbol{e}^{\dagger} \boldsymbol{E}\right]\right)\right\rangle
$$

by

$$
\boldsymbol{\mu}_{p ; q}(\boldsymbol{e})=\left.\left(\frac{2}{i}\right)^{p+q} \nabla_{\boldsymbol{E}}^{\otimes q} \otimes \nabla_{\boldsymbol{E}^{\dagger}}^{\otimes p} \Phi_{\boldsymbol{e}}\right|_{\boldsymbol{E}=0},
$$

where $\boldsymbol{e}^{\dagger} \boldsymbol{E}$ represents an inner product and $\nabla_{\boldsymbol{E}}^{\otimes q}$ is the $q^{\text {th }}$-order gradient with respect to $\boldsymbol{E}$. Also as for scalar fields, the secondary characteristic function

$$
\Psi_{\boldsymbol{e}}(\boldsymbol{E}) \equiv \log \left[\Phi_{\boldsymbol{e}}(\boldsymbol{E})\right]
$$

is used to define the $n^{\text {th }}$-order tensor cumulants

$$
\kappa_{p ; q}(\boldsymbol{e})=\left.\left(\frac{2}{i}\right)^{p+q} \nabla_{\boldsymbol{E}}^{\otimes q} \otimes \nabla_{\boldsymbol{E}^{\dagger}}^{\otimes p} \Psi_{\boldsymbol{e}}\right|_{\boldsymbol{E}=0} .
$$

2 There is a typographical error in Equation 16 of Rickett (1975); the third term on the right-hand side of this equation should be $\left\langle r_{x^{*}}(\tau)\right\rangle\left\langle r_{N}(\tau)\right\rangle$.
The fourth-order cumulants are related to the fourthand second-order moments by (Cardoso 1991) $)^{3}$

$$
\boldsymbol{\kappa}_{2 ; 2}(\boldsymbol{e})=\langle\boldsymbol{r} \otimes \boldsymbol{r}\rangle-\boldsymbol{\rho} \otimes \boldsymbol{\rho}-\boldsymbol{\rho} \tilde{\otimes} \boldsymbol{\rho},
$$

where $\boldsymbol{r} \equiv \boldsymbol{e} \otimes \boldsymbol{e}^{\dagger}$ is the instantaneous coherency matrix, and

$$
\boldsymbol{\rho} \equiv \boldsymbol{\mu}_{1 ; 1}(\boldsymbol{e})=\langle\boldsymbol{r}\rangle
$$

defines the population mean coherency matrix that is typically used to describe the polarization of electromagnetic radiation (Born \& Wolf 1970). The $\tilde{\otimes}$ operator represents a tensor product followed by a transpose over contravariant tensor indeces; i.e.

$$
\begin{aligned}
& \{\mathbf{A} \otimes \mathbf{B}\}_{i k}^{j l} \equiv A_{i}^{j} B_{k}^{l} \\
& \{\mathbf{A} \tilde{\otimes} \mathbf{B}\}_{i k}^{j l} \equiv A_{i}^{l} B_{k}^{j}
\end{aligned}
$$

where $\mathbf{A}$ and $\mathbf{B}$ are rank 2 tensors (i.e. matrices). Appendix $\mathrm{B}$ describes some minor differences between the index notation used in the above definitions and the convention used by Cardoso (1991).

Equations (13) through (16) form the mathematical basis from which the covariances between the Stokes parameters are derived in the following sections. First, the above tensor products are transformed to be expressed in terms of the Stokes parameters associated with matrices $\mathbf{A}$ and $\mathbf{B}$. These transformations are then used to express Equation (13) in terms of the instantaneous and population mean Stokes parameters of a single source of radiation. Finally, in Section 3.2, these tensors are used to derive the covariances between the Stokes parameters in the case of superposed modes of emission represented by $\mathbf{A}$ and $\mathbf{B}$.

\section{COVARIANCES BETWEEN THE INSTANTANEOUS STOKES PARAMETERS}

Although more elegant than its alternative representations, the complex-valued rank 4 tensor of cumulants defined in Equation (13) is not immediately amenable to interpretation. It remains to transform the equations that describe fourth-order tensor products of the electric field vector into the equivalent objects that describe second-order tensor products of the Stokes parameters. This is achieved in the following sections by first identifying the isomorphism between rank 4 tensors in the two-dimensional vector space of the electric field $\mathbb{C}_{2 ; 2}^{2}$ and rank 2 tensors in the four-dimensional vector space of the Stokes parameters $\mathbb{C}_{1 ; 1}^{4}$.

\footnotetext{
3 Section 5.2 of Amblard et al. (1996) concludes with an incorrect expression for this relationship.
} 


\subsection{Isomorphism between $\mathbb{C}_{2 ; 2}^{2}$ and $\mathbb{C}_{1 ; 1}^{4}$}

The required mathematical mapping between fourthorder products of $\boldsymbol{e}$ and second-order products of Stokes parameters derives from the isomorphism between the $2 \times 2$ vector space of the coherency matrix and the fourdimensional vector space of the Stokes parameters, as expressed by the following pair of equations.

$$
\begin{aligned}
\boldsymbol{\rho} & =S_{\mu} \boldsymbol{\sigma}_{\mu} / 2 \\
S_{\mu} & =\boldsymbol{\sigma}_{\mu}: \boldsymbol{\rho}
\end{aligned}
$$

Here, $S_{\mu}$ are the four Stokes parameters, Einstein notation is used to imply a sum over repeated indeces, $0 \leq \mu \leq 3, \sigma_{0}$ is the $2 \times 2$ identity matrix, $\boldsymbol{\sigma}_{1-3}$ are the Pauli matrices, and the : operator represents tensor double contraction, a tensor product followed by contraction over two pairs of indeces. The double contraction of two matrices $\mathbf{A}$ and $\mathbf{B}$ yields a scalar quantity defined by

$$
\mathbf{A}: \mathbf{B} \equiv A_{i}^{j} B_{j}^{i}=\operatorname{Tr}[\mathbf{A B}]
$$

where $\operatorname{Tr}$ is the matrix trace operator. Equation (17) expresses the coherency matrix as a linear combination of Hermitian basis matrices; Equation (18) represents the Stokes parameters as the projections of the coherency matrix onto the basis matrices. When $\boldsymbol{\rho}$ is Hermitian, the Stokes parameters are real-valued.

Using Equations (17) and (18), any linear transformation of the coherency matrix $\boldsymbol{\rho}^{\prime}=L(\boldsymbol{\rho})$, can be expressed as an equivalent linear transformation of the associated Stokes parameters by the Mueller matrix M, as defined by

$$
S_{\mu}^{\prime}=M_{\mu}^{\nu} S_{\nu}=\frac{1}{2} \sigma_{\mu}: L\left(\sigma_{\nu}\right) S_{\nu}
$$

If, for any positive-definite Hermitian matrix $\boldsymbol{\rho}$, the result of the linear transformation $L(\boldsymbol{\rho})$ is also positivedefinite and Hermitian, then $L$ is called positive and its associated Mueller matrix is real-valued. In general, the Mueller matrix may be complex-valued.

Let $L_{\mathbf{U}}(\boldsymbol{\rho})=\mathbf{U}: \boldsymbol{\rho}$ represent the linear transformation of $\boldsymbol{\rho}$ by a rank 4 tensor $\mathbf{U}$ with 2 covariant and 2 contravariant indeces, such that the double contraction yields a matrix with components given by

$$
\{\mathbf{U}: \boldsymbol{\rho}\}_{i}^{j} \equiv U_{i k}^{j l} \rho_{l}^{k} .
$$

Substitution of $L_{\mathbf{U}}$ into Equation (20), followed by elimination of $S_{\nu}$, associates with $\mathbf{U}$ a $4 \times 4$ Mueller matrix,

$$
M_{\mu}^{\nu}=\frac{1}{2} \sigma_{\mu}: \mathbf{U}: \sigma_{\nu}
$$

Likewise, for any $4 \times 4$ Mueller matrix, there is an associated rank 4 tensor,

$$
\mathbf{U}=\frac{1}{2} M_{\mu}^{\nu} \boldsymbol{\sigma}_{\mu} \otimes \boldsymbol{\sigma}_{\nu}
$$

Equation (22) expresses the components of a Mueller matrix as the double projections of a rank 4 tensor $\mathbf{U}$ onto the Hermitian basis matrices. Equation (23) represents $\mathbf{U}$ as a linear combination of the 16 basis tensors formed by all possible tensor products of the 4 Hermitian basis matrices. Combined, these equations establish the required isomorphism between $\mathbb{C}_{2 ; 2}^{2}$ and $\mathbb{C}_{1 ; 1}^{4}$. A similar mapping between a Mueller matrix and its associated target coherency matrix was derived by Cloude (1986) using Kronecker products of the Hermitian basis matrices and the matrix trace operator.

\subsection{Tensor products of the Stokes parameters}

Although Equations (22) and (23) are derived by considering equivalent linear transformations of the coherency matrix and Stokes four-vector, they can be used to map any object from $\mathbb{C}_{2 ; 2}^{2}$ to $\mathbb{C}_{1 ; 1}^{4}$ (and vice versa). Bearing in mind the objective to convert Equation (13) into an equivalent form that is expressed in terms of the Stokes parameters, Equation (22) is first used to convert the tensor products $\mathbf{A} \otimes \mathbf{B} \in \mathbb{C}_{2 ; 2}^{2}$ and $\mathbf{A} \tilde{\otimes} \mathbf{B} \in \mathbb{C}_{2 ; 2}^{2}$ defined by Equations (15) and (16) into the equivalent tensor products $A \otimes B \in \mathbb{C}_{1 ; 1}^{4}$ and $A \tilde{\otimes} B \in \mathbb{C}_{1 ; 1}^{4}$, respectively, where $A$ and $B$ are the Stokes parameters associated with $\mathbf{A}$ and $\mathbf{B}$. Setting either $\mathbf{U}=\mathbf{A} \otimes \mathbf{B}$ or $\mathbf{U}=\mathbf{A} \tilde{\otimes} \mathbf{B}$ in Equation (22), the following transformation properties (Cardoso 1991)

$$
\begin{aligned}
& (\mathbf{A} \otimes \mathbf{B}): \mathbf{C}=\mathbf{A}(\mathbf{C}: \mathbf{B}) \\
& (\mathbf{A} \tilde{\otimes} \mathbf{B}): \mathbf{C}=\mathbf{A C B}
\end{aligned}
$$

are applied to yield

$$
\begin{aligned}
\left\{\mathbf{M}_{\otimes}(\mathbf{A}, \mathbf{B})\right\}_{\mu}^{\nu} & =\frac{1}{2}\left(\boldsymbol{\sigma}_{\mu}: \mathbf{A}\right)\left(\boldsymbol{\sigma}_{\nu}: \mathbf{B}\right) \\
\left\{\mathbf{M}_{\tilde{\otimes}}(\mathbf{A}, \mathbf{B})\right\}_{\mu}^{\nu} & =\frac{1}{2} \boldsymbol{\sigma}_{\mu}:\left(\mathbf{A} \boldsymbol{\sigma}_{\nu} \mathbf{B}\right)
\end{aligned}
$$

Using Equation (18), Equation (26) is related to the tensor product of the Stokes parameters; i.e.

$$
A \otimes B=2 \mathbf{M}_{\otimes}(A, B)
$$

where

$$
\{A \otimes B\}_{\mu}^{\nu} \equiv A_{\mu} B_{\nu} .
$$

Similarly, as shown in Appendix C, Equation (27) yields

$$
A \tilde{\otimes} B=\frac{1}{2}(A \otimes B+B \otimes A-\boldsymbol{\eta} A \cdot B+i A \wedge B),
$$

where $\boldsymbol{\eta}$ is the Minkowski metric tensor with signature $(+,-,-,-)$,

$$
A \cdot B \equiv \eta^{\mu \nu} A_{\mu} B_{\nu}=A_{0} B_{0}-\boldsymbol{A} \cdot \boldsymbol{B}
$$

is the covariant inner product of $A$ and $B$, and $A \wedge B$ is the anti-symmetric covariant exterior product of the Stokes parameters, defined in Equation (56). 


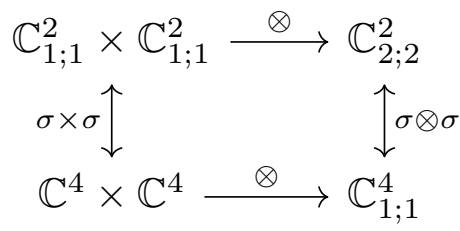

Figure 1. Commutative diagram representing the linear and bilinear mappings between the vector spaces used in this work. In this diagram, $\mathbb{C}_{1 ; 1}^{2}$ represents the vector space of two-dimensional rank 2 tensors such as the coherency matrix and $\mathbb{C}^{4}$ represents the vector space of four-dimensional vectors such as the Stokes parameters. The linear map $\sigma$ represents the isomorphism between these vector spaces as embodied in Equations (17) and (18). In the top row, $\mathbb{C}_{1 ; 1}^{2} \times \mathbb{C}_{1 ; 1}^{2}$ represents a Cartesian product, the vector space of ordered pairs $(\mathbf{A}, \mathbf{B})$ where $\mathbf{A} \in \mathbb{C}_{1 ; 1}^{2}$ and $\mathbf{B} \in \mathbb{C}_{1 ; 1}^{2}$; the tensor product defined by Equation (15) maps these ordered pairs into rank four tensors $\mathbf{A} \otimes \mathbf{B} \in \mathbb{C}_{2 ; 2}^{2}$. Similarly, in the bottom row, $\mathbb{C}^{4} \times \mathbb{C}^{4}$ represents the vector space of ordered pairs $(A, B)$ where $A \in \mathbb{C}^{4}$ and $B \in \mathbb{C}^{4}$; the tensor product defined by Equation (28) maps these ordered pairs into rank 2 tensors $A \otimes B \in \mathbb{C}_{1 ; 1}^{4}$. On the left edge, $\sigma \times \sigma$ represents the Cartesian product of the $\sigma$ operator with itself. When $\sigma \times \sigma$ is applied to an ordered pair, it produces a new ordered pair in which the $\sigma$ operator has been applied separately to each of the two elements; i.e. $(\sigma \times \sigma)(A, B)=(\sigma A, \sigma B)$. On the right edge, $\sigma \otimes \sigma$ represents the isomorphism between $\mathbb{C}_{1 ; 1}^{4}$ and $\mathbb{C}_{2 ; 2}^{2}$, as embodied in Equations (22) and (23). When $\sigma \otimes \sigma$ is applied to a tensor product it produces a new tensor product in which the $\sigma$ operator has been applied separately to each of the two operands; i.e. $(\sigma \otimes \sigma)(A \otimes B)=(\sigma A) \otimes(\sigma B)$. Owing to the universal property of the tensor product, the $\sigma \otimes \sigma$ operator is unique. A similar commutative diagram can be drawn in which the $\otimes$ operators on the top and bottom edges are replaced by the $\tilde{\otimes}$ operator.

Equations (28) and (30) are the required tensor products of the Stokes parameters that yield $4 \times 4$ matrix representations of the tensor products defined by Equations (15) and (16), respectively.

\subsection{The Stokes cumulant}

The results of the previous two sub-sections are summarized in the commutative diagram shown in Figure 1. Although the mappings shown in this diagram have been derived and discussed in the context of the Stokes parameters and coherency matrix, they are purely algebraic transformations between vector spaces and are completely independent of any consideration of statistical moments. In this section, these mappings are applied to convert Equation (13) into an equivalent relation between the cumulants and moments of the Stokes parameters. Setting $\mathbf{U}=\boldsymbol{\kappa}_{2 ; 2}(\boldsymbol{e})$ in Equation (22) and converting each of the three terms on the right hand side of Equation (13) using Equations (28) and (30) yields the Stokes cumulant matrix

$$
\mathbf{Q}=\langle s \otimes s\rangle-S \otimes S-S \tilde{\otimes} S,
$$

where lower-case $s$ represents the instantaneous Stokes four-vector with components defined by

$$
s_{\mu} \equiv r: \sigma_{\mu}=e^{\dagger} \sigma_{\mu} e,
$$

and upper-case $S \equiv\langle s\rangle$ represents the population mean Stokes parameters. Noting that $S \wedge S=0$,

$$
S \tilde{\otimes} S=S \otimes S-\frac{1}{2} \eta S^{2},
$$

where $S^{2}$ is the invariant interval of the Stokes fourvector (e.g. Barakat 1963; Stinebring et al. 1984; Britton 2000) defined by

$$
S^{2} \equiv S \cdot S=S_{0}^{2}-|S|^{2} .
$$

Here, the Stokes four-vector is separated into the total intensity $S_{0}$ and the polarization vector, $\boldsymbol{S}=$ $\left(S_{1}, S_{2}, S_{3}\right)$.

The real-valued $4 \times 4$ matrix $\mathbf{Q}$ represents the fourthorder cumulants of the electric field vector as secondorder moments of the instantaneous Stokes parameters; it is used in Section 3.2 to determine the covariances between the Stokes parameters when two sources of radiation are superposed. Even when considering only a single source of radiation, the Stokes cumulant matrix can be used to compute the covariances between the instantaneous Stokes parameters, which are defined by the $4 \times 4$ covariance matrix,

$$
\mathbf{C} \equiv\langle s \otimes s\rangle-S \otimes S .
$$

Substitution of the above into Equation (32) yields

$$
\mathbf{Q}=\mathbf{C}-S \tilde{\otimes} S .
$$

If the components of the electric field are jointly drawn from a circular complex-valued multivariate normal distribution (Goodman 1963), then all cumulants above second order are equal to zero and $\mathbf{C}=S \tilde{\otimes} S$, which is consistent with the results of Brosseau \& Barakat (1992) and van Straten (2009). In this special case, the covariances between the instantaneous Stokes parameters are completely defined by the population mean Stokes parameters. van Straten (2009) argued that $\mathbf{C}=S \tilde{\otimes} S$ regardless of the distribution of the electric field; however, as shown by Equation (32) and discussed in more detail in Section 4.2, this assertion is incorrect and the relation holds only in the special case that the electric field vector is complex circular normal.

\section{COVARIANCES BETWEEN THE SAMPLE MEAN STOKES PARAMETERS}

A statistical description of the sample mean Stokes parameters begins with the definition of a Stokes sample: a finite sample of $n$ instances of the electric field vector from which the sample mean coherency matrix $\bar{\rho}$ and 
sample mean Stokes parameters $\bar{S}$ are computed via

$$
\overline{\boldsymbol{\rho}} \equiv \frac{1}{n} \sum_{i=1}^{n} \boldsymbol{e}_{i} \otimes \boldsymbol{e}_{i}^{\dagger}=\frac{1}{2} \bar{S}_{\mu} \boldsymbol{\sigma}_{\mu} .
$$

Over a population of independent estimates of $\bar{S}$, each drawn from mutually exclusive Stokes samples, ${ }^{4}$ the covariances between the sample mean Stokes parameters are defined by the $4 \times 4$ covariance matrix,

$$
\overline{\mathbf{C}}=\langle\bar{S} \otimes \bar{S}\rangle-S \otimes S,
$$

where $S \equiv\langle\bar{S}\rangle$ represents the population mean Stokes parameters. $^{5}$ If the instances of the electric field vector are statistically independent and identically distributed, then $\overline{\mathbf{C}}=\mathbf{C} n^{-1}$, where $\mathbf{C}$ is the covariance matrix of the instantaneous Stokes parameters defined in Equation (35) and $n$ is the number of independent instances of the electric field in each Stokes sample.

Figure 2 illustrates the special case of Stokes samples drawn in the time domain; however, it should be noted that the analysis presented in this paper applies in any domain (temporal, spectral, or spatial). In addition to emission from a single source (or mode) of radiation, this figure depicts the combination of electromagnetic waves from two sources (or two modes of emission from a single source) in the three special cases outlined in the introduction (disjoint, superposed, and composite samples). These classifications are highly idealized and in reality the distinction between statistical regimes may not be so clean. For example, the scale for switching between mutually exclusive modes could be variable and span intervals that are both smaller and greater than the integration interval of the instrument; in this case, the source would be described by a mixture of disjoint and composite samples. Similarly, mutually exclusive modes can become partially superposed by processes that introduce temporal, spatial, or spectral coherence, such as passage through a linear time-invariant system (such as the interstellar medium) that is characterized by an impulse response function with a duration that exceeds the timescale for switching between modes. Nevertheless, these idealizations provide a sound conceptual framework on which to base more realistic models.

In the following sections, $\boldsymbol{e}_{A}$ and $\boldsymbol{e}_{B}$ are the electric field vectors associated with two modes of emission; the modes are strictly stationary and have population mean Stokes parameters, $A$ and $B$. The two modes may have completely different statistical distributions; e.g. the in-

4 For example, the population could consist of the sample mean Stokes parameters for a given phase bin drawn from a series of sub-integrations of the average pulse profile.

5 The population mean of the sample mean Stokes parameters is equal to the population mean of the instantaneous Stokes parameters.

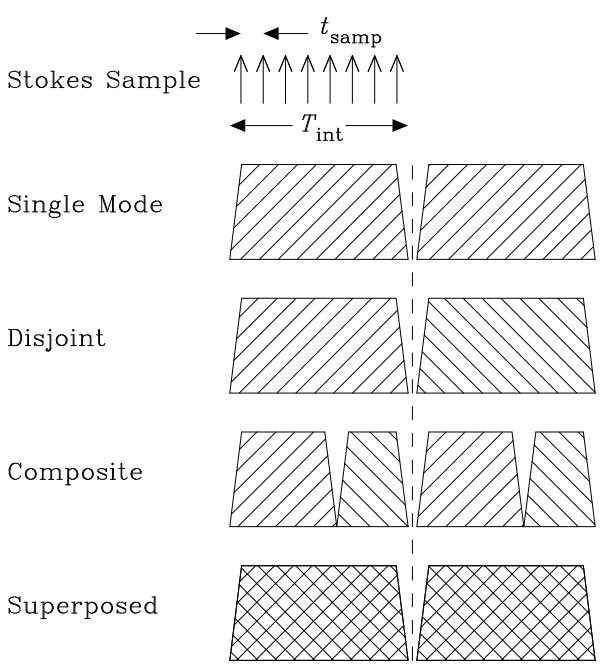

Figure 2. Depiction of different Stokes sample types in the time domain. A Stokes sample (represented as a series of $n=8$ delta functions) consists of a sequence of instances of the electric field sampled at regular intervals separated by $t_{\text {samp }}$ and spanning an integration interval defined by $T_{\text {int }}$. Two independent Stokes samples are shown separated by a dashed vertical line. Emission in one mode is represented by a trapezoid filled with hatch lines of a single orientation; emission in the other mode is represented using hatch lines rotated by 90 degrees. Each disjoint Stokes sample is entirely comprised of instances drawn exclusively from only one population or the other. Composites Stokes samples include instances drawn from both populations. When the modes are superposed, a cross hatch pattern represents the new polarization state that arises when electric field instances drawn from both populations are added together before the instantaneous Stokes parameters are computed. Although two Stokes samples are shown side-by-side, they need not necessarily be contiguous in the time domain. For example, in single-pulse studies, each Stokes sample might be integrated over a fixed range of pulsar longitude such that the start time of each Stokes sample is temporally separated from the next by the pulsar spin period. In this case, the sample mean Stokes parameters record the polarization state of the pulsar emission over that longitude range as a function of pulse number (integer turns of the pulsar).

tensity of mode A could be log-normally distributed and that of mode B could be exponentially distributed. It is assumed that instances of the electric field from a single mode are statistically independent and identically distributed; therefore $\overline{\mathbf{C}}_{A}=\mathbf{C}_{A} n^{-1}$ and $\overline{\mathbf{C}}_{B}=\mathbf{C}_{B} n^{-1}$ describe the covariances between the sample mean Stokes parameters after averaging over a Stokes sample of $n$ instances of the electric field drawn exclusively from either mode A or mode B, respectively. Sections 3.1 through 3.3 present general expressions for the covariances between the Stokes parameters in the three statistical regimes, illustrative examples are presented in Section 3.4, and in Appendix D it is demonstrated that composite samples of unresolved disjoint modes may have been incorrectly identified as superposed modes in previous studies. 


\subsection{Disjoint Samples}

If the modes are mutually exclusive and the interval over which the Stokes parameters are integrated is smaller than the scale over which mode switching occurs, then the modes are resolved. In this case, every instance of the electric field vector in a given Stokes sample is drawn exclusively from only one of the two populations and the sample mean Stokes parameters are given by either $\bar{A}$ or $\bar{B}$. When averaged over many Stokes samples, the population mean Stokes parameters are given by the weighted mean of the population mean Stokes parameters of each mode, $S=F A+(1-F) B$, where $F$ is the fraction of Stokes samples that occur in mode A. Although $F$ might reasonably fluctuate (e.g. as a function of time), only the ideal case in which $F$ remains constant is considered for now. If the modes are statistically independent, then the covariance matrix of the sample mean Stokes parameters is given by

$$
\overline{\mathbf{C}}_{d}=F \overline{\mathbf{C}}_{A}+(1-F) \overline{\mathbf{C}}_{B}+F(1-F)(A-B)^{\otimes 2},
$$

where $(A-B)^{\otimes 2}=(A-B) \otimes(A-B)$ is the second tensor power of the differences between the population mean Stokes parameters of the modes. As shown in Appendix D, the above equation is consistent with the definition of disjoint modes presented in equation (5) of Cordes et al. (1978).

\subsection{Superposed Samples}

When the electromagnetic wave modes are superposed, each instance of the electric field vector in a Stokes sample is given by the sum, $\boldsymbol{e}=\boldsymbol{e}_{A}+\boldsymbol{e}_{B}$. For superposed samples, the population mean coherency matrix is given by

$$
\rho=\rho_{A}+\rho_{B}+\chi+\chi^{\dagger}
$$

where $\boldsymbol{\rho}_{A}$ and $\boldsymbol{\rho}_{B}$ are the coherency matrices of $\boldsymbol{e}_{A}$ and $\boldsymbol{e}_{B}$, and

$$
\chi \equiv\left\langle e_{A} e_{B}^{\dagger}\right\rangle
$$

is the cross-coherency matrix that describes the coherence of the polarized modes. In the special case of an incoherent sum, the population mean $\chi=0$; however, in each finite Stokes sample, the sample mean $\bar{\chi}$ is not exactly zero and the variance of the sample mean crosscoherency contributes to the fourth-order moments of the electric field.

If the modes are statistically independent, then the population mean Stokes parameters are $S=A+B$ and the Stokes cumulant of the sum is $\mathbf{Q}=\mathbf{Q}_{A}+\mathbf{Q}_{B}$. Using Equation (32), the covariances between the sample mean Stokes parameters of the sum are

$$
\overline{\mathbf{C}}_{s}=\overline{\mathbf{C}}_{A}+\overline{\mathbf{C}}_{B}+n^{-1} A \tilde{\odot} B,
$$

where

$$
\begin{aligned}
A \tilde{\odot} B & =A \tilde{\otimes} B+B \tilde{\otimes} A \\
& =A \otimes B+B \otimes A-\boldsymbol{\eta} A \cdot B
\end{aligned}
$$

is twice the symmetric part of $A \tilde{\otimes} B$; it describes the cross-covariance between the instantaneous Stokes parameters of the modes and depends on only the population mean Stokes parameters of the modes. As shown in Appendix D, Equation (42) is consistent with the definition of superposed modes presented in equation (5) of Cordes et al. (1978) only in the special case of $100 \%$ polarized modes and only when considering the instantaneous Stokes parameters.

The cross-covariance $A \widetilde{\odot} B$ is a function of only the population mean Stokes parameters and does not explicitly depend on unique statistical degrees of freedom for each of the modes, as asserted in the erratum of van Straten (2009). As an aside, note that the variance of the instantaneous $(n=1)$ total intensity predicted by the above equation is consistent with the variance of the instantaneous intensity of superposed scalar fields. This can be shown by transforming the electric field vector by a singular Jones matrix that reduces its dimension to that of a single scalar field. In this basis, all sources are observed to be $100 \%$ polarized, such that $A \cdot B=0$ and $\left\{\overline{\mathbf{C}}_{s}\right\}_{0}^{0}$ of Equation (42) reduces to $\varsigma_{\xi}^{2}$ of Equation (7).

\subsection{Composite Samples}

When only one mode contributes at any instant and both modes contribute to the signal in the interval over which the sample mean Stokes parameters are integrated, then the disjoint modes are unresolved. In this case, each Stokes sample is a union of sub-samples drawn from two mutually exclusive populations and the composite sample mean Stokes parameters are equal to the weighted average of the sample mean Stokes parameters of each mode; i.e. $\bar{S}=f \bar{A}+(1-f) \bar{B}$, where $f$ is the constant fraction of samples that occur in mode $\mathrm{A}$ in each Stokes sample. It is also reasonable that $f$ might fluctuate between Stokes samples; here, only the ideal case in which $f$ remains constant is considered. If the modes are statistically independent, then the covariance matrix of the sample mean Stokes parameters is given by

$$
\overline{\mathbf{C}}_{c}=f \overline{\mathbf{C}}_{A}+(1-f) \overline{\mathbf{C}}_{B} .
$$

As shown in Appendix D, the above equation is consistent with the definition of mode superposition presented in equation (5) of Cordes et al. (1978). It is also consistent with the definition of superposed modes described in Section 2.1 of McKinnon \& Stinebring (1998) and with the definition of an incoherent sum described in Section 4.3 of van Straten (2009). In the erratum to the latter work, van Straten (2010) redefines an incoherent 
sum that is consistent with classical wave superposition, as defined in the previous section; however, the distinc- tion between superposed and composite samples was not recognized in this previous work.

Table 1. Regimes of Mode Combination

\begin{tabular}{lccc}
\hline \hline \multicolumn{1}{c}{ Property } & Superposed & Composite & Disjoint \\
\hline Electric Field Instances & $\boldsymbol{e} \in\left\{\boldsymbol{e}_{A}+\boldsymbol{e}_{B}\right\}_{n}$ & $\boldsymbol{e} \in\left\{\boldsymbol{e}_{A}\right\}_{f n} \cup\left\{\boldsymbol{e}_{B}\right\}_{(1-f) n}$ & $\boldsymbol{e} \in\left\{\boldsymbol{e}_{A}\right\}_{n} \underline{\vee} \boldsymbol{e}_{i}\left\{\boldsymbol{e}_{B}\right\}_{n}$ \\
Sample Mean Stokes Parameters & $\bar{S} \in\{\bar{A}+\bar{B}\}_{N}$ & $\bar{S} \in\{f \bar{A}+(1-f) \bar{B}\}_{N}$ & $\bar{S} \in\{\bar{A}\}_{F N} \cup\{\bar{B}\}_{(1-F) N}$ \\
Covariances of Sample Means & $\overline{\mathbf{C}}_{A}+\overline{\mathbf{C}}_{B}+n^{-1} A \tilde{\odot} B$ & $f \overline{\mathbf{C}}_{A}+(1-f) \overline{\mathbf{C}}_{B}$ & $F \overline{\mathbf{C}}_{A}+(1-F) \overline{\mathbf{C}}_{B}+F(1-F)(A-B)^{\otimes 2}$ \\
Incoherent Sum & pre-detection & post-detection & N/A \\
Mutual Exclusivity & N/A & unresolved & resolved \\
\hline
\end{tabular}

\subsection{Illustrative Examples}

The three regimes of mode combination presented in the previous sub-sections are summarized in Table 1 , where $\{\boldsymbol{e}\}_{n} \equiv\left\{\boldsymbol{e}_{1}, \boldsymbol{e}_{2}, \ldots \boldsymbol{e}_{n}\right\}$ is a Stokes sample of $n$ instances of the electric field vector, $\{\bar{S}\}_{N}$ is a population of $N \rightarrow \infty$ instances of sample mean Stokes parameters, $\cup$ is the set union operator, and $\underline{V}$ is the exclusive disjunction. The last two rows of this table highlight some of the semantic and conceptual similarities between the three statistical regimes. The term "incoherent sum" is used to describe both the pre-detection classical wave superposition of statistically independent signals (e.g. astronomical source plus receiver noise) and the postdetection integration of flux densities (e.g. integration over time and radio frequency). A source that switches between mutually exclusive states will result in either composite or disjoint samples, depending on the interval over which the sample mean is integrated and the characteristic scale for mode switching.

To illustrate the fundamental differences between the three types of Stokes sample, consider the incoherent sum of orthogonally polarized modes with normally distributed electric fields, equal population mean intensities $I$ and equal degrees of polarization $p$. Note that orthogonally polarized states have anti-parallel population mean Stokes polarization vectors and, without any loss of generality, assume that the basis in which the Stokes parameters are measured is the natural basis defined by the modes, such that $S_{2}=S_{3}=0$. Furthermore, for both composite and disjoint samples, the modes occur with equal frequency (i.e. $f=F=0.5$ ) such that, for all three sample types, the resulting signal is completely depolarized; i.e. the population mean Stokes polarization vector $\boldsymbol{S}=0$. Finally, assume that each Stokes sample is sufficiently large that the distributions of the sample mean Stokes parameters are well approximated by a multivariate normal distribution. (This is a reasonable assumption in single-pulse studies because each phase bin typically spans thousands of instances of the electric field.) Note that the population mean Stokes parameters provide no information about the manner in which the signals have been combined. However, as depicted in Figure 3, the three-dimensional distributions of the sample mean Stokes polarization vector are fundamentally different in each case.

These differences are also evident in the structure of the $4 \times 4$ matrix of covariances between the sample mean Stokes parameters. When only a single source contributes, $\mathbf{C}=S \tilde{\otimes} S$ and in the natural basis Equation (33) yields

$$
\overline{\mathbf{C}}_{\text {single }}=\frac{1}{2 n}\left(\begin{array}{cccc}
\|S\|^{2} & 2 I^{2} p & 0 & 0 \\
2 I^{2} p & \|S\|^{2} & 0 & 0 \\
0 & 0 & S^{2} & 0 \\
0 & 0 & 0 & S^{2}
\end{array}\right),
$$

where the Euclidean norm

$$
\|S\|^{2} \equiv S_{0}^{2}+|S|^{2} .
$$

In this example, $\|S\|^{2}=I^{2}\left(1+p^{2}\right)$ and $S^{2}=I^{2}\left(1-p^{2}\right)$. Note that the principal polarization $S_{1}$ is covariant with the total intensity $S_{0}$ and that the variances of $S_{0}$ and $S_{1}$ are equal. Furthermore, the variances of $S_{2}$ and $S_{3}$ are equal and less than the variances of $S_{0}$ and $S_{1}$; therefore, the distribution of the sample mean Stokes polarization vector is described by a prolate spheroid that is rotationally symmetric about the population mean Stokes polarization vector. The axial ratio of this spheroid, $\epsilon=\left(\left(1+p^{2}\right) /\left(1-p^{2}\right)\right)^{1 / 2}$, is completely defined by the degree of polarization of the population mean Stokes parameters. 
For disjoint samples, the bimodal distribution of sample mean Stokes parameters is no longer accurately described by a multivariate normal distribution. The difference between the population mean polarization vector of each mode (i.e. the distance between the centres of each prolate spheroid, $2 I p$ ) adds to the estimated variance along the principal axis. This is readily seen by expressing Equation (39) in the natural basis, which yields

$$
\overline{\mathbf{C}}_{\text {disjoint }}=\frac{1}{2 n}\left(\begin{array}{cccc}
\|S\|^{2} & 0 & 0 & 0 \\
0 & \|S\|^{2}+2 n I^{2} p^{2} & 0 & 0 \\
0 & 0 & S^{2} & 0 \\
0 & 0 & 0 & S^{2}
\end{array}\right)
$$

Whereas the sizes of the disjoint prolate spheroids are inversely proportional to the square root of the sample size, the distance between their centres is not decreased by integration.

For superposed samples, the instantaneous intensity is doubled and the cross-covariance between the modes yields a hyperspherically symmetric distribution described by Equation (42),

$$
\overline{\mathbf{C}}_{\text {superposed }}=\frac{1}{2 n}\left(\begin{array}{cccc}
4 I^{2} & 0 & 0 & 0 \\
0 & 4 I^{2} & 0 & 0 \\
0 & 0 & 4 I^{2} & 0 \\
0 & 0 & 0 & 4 I^{2}
\end{array}\right) .
$$

In this example, superposition yields unpolarized radiation, for which the distribution of sample mean Stokes parameters is expected to be hyperspherically symmetric when the voltage is normally distributed.

For composite samples, integration over an equal number of instances of each mode yields a prolate spheroidal distribution of the sample mean Stokes polarization vector with the same dimensions as those of the original modes, now centred on the origin; i.e., from Equation (44),

$$
\overline{\mathbf{C}}_{\text {composite }}=\frac{1}{2 n}\left(\begin{array}{cccc}
\|S\|^{2} & 0 & 0 & 0 \\
0 & \|S\|^{2} & 0 & 0 \\
0 & 0 & S^{2} & 0 \\
0 & 0 & 0 & S^{2}
\end{array}\right) .
$$

In this example, when orthogonal modes are combined, the principal polarization is no longer covariant with the total intensity (i.e. $C_{0}^{1}=C_{1}^{0}=0$ ) because the two modes contribute equally and oppositely to this term. For both disjoint and composite samples, the axial ratio $\epsilon=\varsigma_{1} / \varsigma_{2}$ that is inferred from the covariance matrix is greater than unity. As this is inconsistent with the

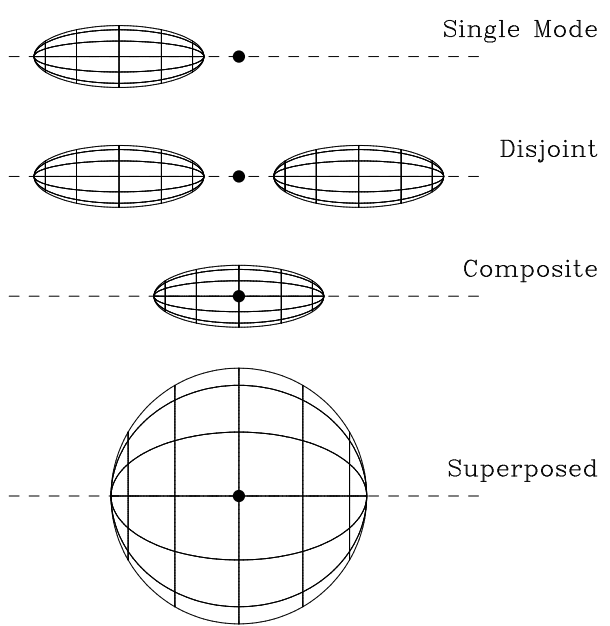

Figure 3. Distributions of the sample mean Stokes polarization vector in each regime of orthogonal mode combination. In each row, the origin (unpolarized flux) is marked by a dot and the principal axis (defined by the population mean polarization vectors of the modes) is marked by the dashed line that runs horizontally through the origin. All of the spheroids are symmetric under rotation about the principal axis, the axes of the spheroids are proportional to the standard deviations of the components of the sample mean Stokes polarization vector $\overline{\boldsymbol{S}}=\left(\bar{S}_{1}, \bar{S}_{2}, \bar{S}_{3}\right)$, and the axial ratios are defined by the degree of polarization of the population mean Stokes parameters, $p=|\boldsymbol{S}| / S_{0}$.

degree of polarization of the population mean Stokes parameters, it potentially serves as an experimental indicator of mutually exclusive modes.

\section{INTERPRETATION}

Given only the population mean Stokes parameters, the three regimes of mode combination (disjoint, superposed, and composite samples) described in the previous section cannot be distinguished; however, in principle, they may be differentiated via the covariances between the Stokes parameters. A $4 \times 4$ symmetric and realvalued covariance matrix contains 10 unique elements; using principal component analysis, these may be reduced to 7 numbers of interest as follows. First, conformably partition the $4 \times 4$ covariance matrix into the variance of the total intensity, the $3 \times 3$ covariance matrix of the Stokes polarization vector, and the 3 -dimensional vector of covariances between the total intensity and the components of the polarization vector. Then, project the Stokes parameters onto the basis defined by the eigen decomposition of the $3 \times 3$ covariance matrix of the Stokes polarization vector. This diagonalizes the $3 \times 3$ partition, leaving only four variances $\varsigma_{\mu}^{2}=C_{\mu}^{\mu}$ along the diagonal and three covariances $C_{0}^{j}\left(=C_{j}^{0}\right)$ in the first row (and column). In this eigenbasis, assuming normally distributed electric field components, the following conditions hold. 
1. Both a single mode and superposed modes produce prolate spheroidal distributions of the sample mean Stokes polarization vector such that the primary axis of the distribution (as defined by the eigenvector with the largest eigenvalue) is aligned with the population mean Stokes polarization vector, the distribution is symmetric under rotation about this axis $\left(\varsigma_{2}=\varsigma_{3}\right)$, and the axial ratio $\epsilon \equiv \varsigma_{1} / \varsigma_{2}=\left(\left(1+p^{2}\right) /\left(1-p^{2}\right)\right)^{1 / 2}$, where $p=|\boldsymbol{S}| / S_{0}$ is the degree of polarization of the population mean Stokes parameters. The standard deviation of the primary polarization is equal to that of the total intensity (i.e. $\varsigma_{1}=\varsigma_{0}$ ) and the total intensity is uncorrelated with the minor polarizations (i.e. $C_{0}^{2}=C_{0}^{3}=0$ ).

2. Mutually exclusive orthogonally polarized modes (both disjoint and composite samples) also exhibit prolate spheroidal distributions of the sample mean polarization vector with cylindrical symmetry about the population mean polarization vector. However, in this case, the axial ratio is inconsistent with the degree of polarization of the population mean Stokes parameters.

3 . In the case of disjoint modes, the standard deviation of the primary polarization $\varsigma_{1}$ may exceed the standard deviation of the total intensity $\varsigma_{0}$.

However, several important phenomena invalidate the above simple observations. First, as described in more detail in Section 4.1, the contribution of superposed noise from both the sky and the instrument must be properly subtracted before the covariances between the Stokes parameters that are intrinsic to the source can be interpreted. Second, the equations that describe superposed and composite samples are valid only when the modes are statistically independent. Any dependence between the modes, such as partial coherence or covariant intensities, will invalidate points 1 and 3 above. Partial coherence of orthogonally polarized modes (e.g. Gangadhara 1997) is a plausible means of generating non-orthogonal modes and, in the case of composite samples, covariant mode intensities cause the variance of the primary polarization to differ from that of the total intensity (e.g. McKinnon \& Stinebring 1998). In particular, anticorrelated mode intensities can mimic the observable effects of disjoint modes by causing the variance of the primary polarization to be greater than that of the total intensity. Third, the effects of propagation through inhomogeneities in the electron density (e.g. Cordes et al. 2004) and magnetic field (e.g. Melrose \& Macquart 1998) of the interstellar medium have not been considered. Finally, as described in more detail in Section 4.2, amplitude modulation alters the form of the covariance matrix in a manner that depends on the correlated structure of the amplitude modulating function. To distinguish between pulsar-intrinsic and scintillation-induced variability, future work will incorporate analyses based on the autocorrelation functions of the Stokes parameters (e.g. Cordes \& Hankins 1977; Cordes et al. 2004) and may employ techniques similar to the fluctuation spectral analysis introduced by Edwards \& Stappers (2003); Edwards (2004); Edwards \& Stappers (2004).

\subsection{Superposed Sky and Instrumental Noise}

Consider the observation of a source of electromagnetic radiation described by the population mean Stokes parameters $S_{\mathrm{S}}$ and covariance matrix $\mathbf{C}_{\mathrm{S}}$ superposed with statistically independent sky and instrumental noise described by population mean Stokes parameters $S_{\mathrm{N}}$ and covariance matrix $\mathbf{C}_{\mathrm{N}}$. Section 4.3 of van Straten (2009) incorrectly asserts that the incoherent addition of unpolarized noise adds a constant term to each element of the diagonal of the observed covariance matrix. Rather, using Equation (42), the covariance matrix of the observed superposition is given by

$$
\mathbf{C}_{\mathrm{obs}}=\mathbf{C}_{\mathrm{S}}+\mathbf{C}_{\mathrm{N}}+S_{\mathrm{S}} \odot S_{\mathrm{N}}
$$

The above equation requires no assumptions regarding the distribution of the electric field or the nature of the source (e.g. mode switching). It is more generally applicable than Equation (9) of Cordes \& Hankins (1977), which is valid only under the assumption that the system and sky noise are unpolarized and normally distributed. To solve Equation (47) for the source-intrinsic covariance matrix $\mathbf{C}_{\mathrm{S}}$, it is necessary to subtract both $\mathbf{C}_{\mathrm{N}}$ and $S_{\mathrm{S}} \odot S_{\mathrm{N}}$ from the observed covariance matrix. In observations of radio pulsars, both $S_{\mathrm{N}}$ and $\mathbf{C}_{\mathrm{N}}$ are readily obtained from the off-pulse noise statistics. However, if it can be assumed that the electric field of the noise is normally distributed, then greater sensitivity is achieved by estimating the covariances between the noise Stokes parameters via $\mathbf{C}_{\mathrm{N}}=S_{\mathrm{N}} \tilde{\otimes} S_{\mathrm{N}}$. The Stokes parameters of the source $S_{\mathrm{S}}$ are obtained by subtracting $S_{\mathrm{N}}$ from the observed Stokes parameters $S$. Given this information, Equation (47) is trivial to solve for $\mathbf{C}_{\mathbf{S}}$.

\subsection{Amplitude Modulation}

The derivation of the covariances between the sample mean Stokes parameters presented in Section 3.3 of van Straten (2009) begins with the incorrect assertion that, for unpolarized radiation, the covariance matrix $\mathbf{C}$ is proportional to the $4 \times 4$ identity matrix, regardless of the distribution of the electric field vector. This assertion derives from the erroneous presumption that uncorrelated signals are also statistically independent. ${ }^{6}$

6 The faulty reasoning proceeds as follows. First, because the radiation is unpolarized, the instantaneous intensities of the uncorrelated electric field vector components must also be uncorrelated; therefore, because $S_{0}$ and $S_{1}$ are the sums and differences 
This flawed reasoning is also reflected in Section 4.1 of van Straten (2009), in which it is incorrectly argued that scalar amplitude modulation uniformly scales the covariances of the Stokes parameters by reducing the effective statistical degrees of freedom.

In fact, scalar amplitude modulation introduces statistical dependence between the components of the electric field vector, such that their instantaneous intensities are correlated. As the total intensity is the sum of the intensities of the two field components, positive covariance between the field component intensities increases the variance of the amplitude-modulated total intensity, even in the case of unpolarized radiation. In contrast, each of the components of the Stokes polarization vector can be represented as differences in the intensities of the electric field components in a given basis (Born \& Wolf 1970). Therefore, positive covariance between the field component intensities decreases the variances of the amplitude-modulated Stokes polarization vector components. That is, when the electric field vector is amplitude modulated by a scalar multiplier, the variances of the components of the Stokes polarization vector are expected to be less than the variance of the total intensity.

The above reasoning is formally demonstrated by considering amplitude modulation of the electric field vector by a statistically independent random and real-valued dimensionless scalar $\sqrt{u}$, producing $e^{\prime}=\sqrt{u} \boldsymbol{e}$. Using a variation of the technique introduced by Goodman (1960), the covariances between the modulated instantaneous Stokes parameters are shown to be

$$
\mathbf{C}^{\prime}=\left(\varsigma_{u}^{2}+1\right) \mathbf{C}+\varsigma_{u}^{2} S \otimes S,
$$

where $\varsigma_{u}^{2}$ is the variance of $u, \mathbf{C}$ is the covariance matrix of the unmodulated instantaneous Stokes parameters, $S$ is the population mean Stokes four-vector, and without any loss of generality it is assumed that scalar amplitude modulation does not alter the population mean Stokes parameters (i.e. $\langle u\rangle=1$ ). In the natural basis where $S_{0} \geq S_{1} \geq 0$ and $S_{2}=S_{3}=0$, it is readily seen that the last term in the right-hand side of Equation (48) increases only the variances of the total intensity $S_{0}$ and the primary polarization $S_{1}$ and the covariance be-

of uncorrelated random values, their variances should be equal. Second, the statistics of unpolarized radiation should be independent of the basis in which it is measured; therefore, the variances of all three components of the Stokes polarization vector should be equal. A simple counter-example disproves both of these assumptions. Consider an electric field vector with components that have random, uncorrelated phases but equal amplitudes given by the random variate $\sqrt{a}$. The resulting signal is completely unpolarized; however, the field component intensities are completely correlated. In this case, the variance of $S_{0}$ is four times the variance of $a$, the variance of $S_{1}$ is zero, and the variances of $S_{2}$ and $S_{3}$ are equal to the second moment of $a$. tween them. Furthermore, for partially polarized radiation where $S_{0}>S_{1}$, amplitude modulation increases the variance of the total intensity $\varsigma_{0}^{2}$ by more than it increases the variance of the primary polarization $\varsigma_{1}^{2}$. This provides a simpler explanation for the observation made in Section 4.4 of van Straten (2009), in which it is argued that orthogonally polarized modes with covariant intensities could explain $\varsigma_{0}>\varsigma_{1}$. Rather, amplitude modulation causes $\varsigma_{0}>\varsigma_{1}$ even when only a single mode of emission contributes to the observed signal.

Equation (48) is valid only for the instantaneous Stokes parameters. To derive the covariances between the sample mean Stokes parameters, any correlations between instances of the amplitude modulating scalar variate (e.g. subpulse structure) must be considered, which is beyond the scope of this paper. Temporally correlated structure of the amplitude modulation function has been rigorously studied in the seminal works of Rickett (1975) and Cordes (1976).

\section{CONCLUSION}

The statistical framework presented in this paper can be applied to sources with arbitrary distributions of flux density; it also incorporates the effects of instrumental integration over finite samples. Therefore, it is ideally suited to the study of variability in polarized radiation on short timescales, such as the polarization of subpulse structure in radio pulsar emission.

Consideration of integration over finite samples highlights the following important results. First, the arguments that have been presented to date in support of superposed modes of pulsar radiation apply equally well to a composite sample of unresolved disjoint modes. For example, both superposition and composition of modes result in depolarization when integrated over a finite sample. As argued in the introduction and elaborated in Appendix A, observational evidence of unresolved disjoint modes has already been presented in the published literature. In Appendix D, the statistical test defined by Equation (5) of CRB is shown to be valid only when the orthogonally polarized modes are assumed to be $100 \%$ polarized and only when the second moments of the instantaneous Stokes parameters are measured. When this test is applied to single-pulse data comprised of sample mean Stokes parameters, a composite sample of mutually exclusive modes will be incorrectly identified as superposed.

In principle, it is possible to differentiate between the three regimes of mode combination (disjoint, superposed, and composite samples) through analysis of the covariances between all four Stokes parameters. However, various physical phenomena complicate interpretation. Most importantly, amplitude modulation - primarily that intrinsic to the pulsar emission mechanism, 
but also that arising in the interstellar medium - significantly alters the structure of the $4 \times 4$ covariance matrix. The impact of amplitude modulation on the covariances between the sample mean Stokes parameters depends on the correlated structure of the amplitude modulating function (e.g. subpulse structure). More detailed treatments of phenomena such as amplitude modulation (e.g. Osłowski et al. 2013), partial mode coherence (e.g. Gangadhara 1997), covariant mode intensities (e.g. McKinnon \& Stinebring 1998), and scintillation in a magnetized plasma (e.g. Melrose 1993a,b) are required before the statistical framework presented in this paper will be sufficiently developed to interpret observational data. Therefore, a set of simulations were developed to verify the equations presented in this work; these are described in Appendix E.

Parts of this research were supported by the Australian Research Council Centre of Excellence for Allsky Astrophysics (CAASTRO), through project number CE110001020, and the Australian Laureate Fellowships scheme, through project number FL150100148. The authors are grateful to Pablo Rosado for helping to test the derivation presented in Appendix C. We also thank Damien Hicks for useful discussions and feedback on the paper.

\section{APPENDIX}

\section{A. EVIDENCE FOR DISJOINT MODES OBSERVED BY GANGADHARA ET AL. (1999)}

The scatter plots presented in Figure 3 of Gangadhara et al. (1999), hereafter G+99, demonstrate that the degree of polarization of single pulses (both fractional linear and fractional circular polarization) increases as the instrumental integration length (or sample size) is decreased. To support the argument that this correlation represents plausible evidence of unresolved, disjoint, and orthogonally polarized modes, it is necessary to rule out a comparably plausible alternative interpretation: the observed correlation between temporal resolution and degree of polarization could be a manifestation of a well known statistical bias to the degree of polarization that increases as the sample size is decreased; e.g. see Figure 3 of van Straten (2009), hereafter vS09.

G+99 observed PSR B1133+16 using an instrument with a bandwidth of $\Delta \nu=40 \mathrm{MHz}$ and, even on the shortest time scale presented, $\tau_{\min }=150 \mu \mathrm{s}$, the sample size (given by the time-bandwidth product) is $n=\tau_{\min } \Delta \nu=6 \times 10^{3}$. For normally distributed noise, Equation (B4) of vS09 predicts a maximum expected bias to the degree of polarization of less than $2 \%$, which is too small to explain the correlation observed by G+99. However, as discussed in Section 4 of vS09, the effective sample size (or statistical degrees of freedom) may be reduced by amplitude modulation and wave coherence, such as that introduced by multipath propagation in the interstellar medium. The scintillation bandwidth of PSR B1133+16 is around $60 \mathrm{MHz}$ at $1 \mathrm{GHz}$ (Cordes 1986) and the corresponding coherence time in the observations by $\mathrm{G}+99$ (made at a center frequency of $1.41 \mathrm{GHz}$ ) is 4 to 5 orders of magnitude smaller than $\tau_{\text {min }}$. Therefore, the impact of scattering on the effective sample size is negligible.

To estimate the order of magnitude of the bias to the degree of polarization induced by amplitude modulation, it is necessary to characterize and model the physical nature of subpulse structure, which varies between pulsars. For the purposes of this argument, it suffices to describe the subpulse structure by its characteristic width $\tau$ and the modulation index $\beta$ that is observed after the instrument integrates over some number of unresolved subpulses. Conservative selection of these parameters for PSR B1133+16 is based on the following derivation of the relationship between them.

Consider normally distributed unpolarized noise that is modulated by a contiguous sequence of rectangular subpulses. Assume that the unpolarized noise has a population mean total intensity of unity and that the characteristic subpulse width $\tau$ corresponds to a sub-sample size $n^{\prime}$; therefore, before modulation, the sub-sample mean total intensity $\bar{S}_{0}^{\prime}$ has a variance of $\left(2 n^{\prime}\right)^{-1}$, as given by Equation (45). As noted in the introduction, radio pulsar emission typically exhibits log-normal distributions of subpulse amplitude (e.g. Cairns et al. 2003; Osłowski et al. 2014). Therefore, let the amplitude $u$ of each rectangular subpulse be drawn from a log-normal distribution; i.e. $u=\exp (v)$, where $v$ is a normally distributed random variate with zero mean and standard deviation $\varsigma$, such that the mean and variance of $u$ are $\langle u\rangle=\exp \left(\varsigma^{2} / 2\right)$ and $\varsigma_{u}^{2}=\exp \left(2 \varsigma^{2}\right)-\exp \left(\varsigma^{2}\right)$ respectively.

Now consider the average of $N^{\prime}=n / n^{\prime}$ such subpulses, yielding the sample mean of $N^{\prime}$ statistically independent instances of the amplitude modulated sub-sample mean intensity, $u \bar{S}_{0}^{\prime}$. The variance of the sample mean normalized by the square of the population mean yields the square of the modulation index

$$
\beta^{2}=\frac{1}{n}\left[\left(\exp \left(\varsigma^{2}\right)-1\right)\left(n^{\prime}+\frac{1}{2}\right)+\frac{1}{2}\right],
$$


which is trivially solved for the value of $\varsigma$ required to yield a chosen modulation index, given the sample size $n$ and the sub-sample size $n^{\prime}$ of the modulating subpulses.

For a fixed sample size, Equation (49) shows that the modulation index decreases as the subpulse width decreases, which necessitates a larger value of $\varsigma$ to achieve a certain sample mean intensity modulation index. In turn, this decreases the effective sample size and increases the bias to the degree of polarization. Therefore, to present a conservative upper limit on bias, the shortest microstructure characteristic timescale, $\tau_{\mu-\text { narrow }} \sim 10 \mu$ s, estimated by Popov et al. (2002) is adopted. Similarly, from the phase-resolved modulation index of PSR B1133+16 presented in Figure A.19 of Weltevrede et al. (2006), the maximum value of $\beta \sim 2.4$ is assumed. The adopted values yield $n^{\prime}=\tau_{\mu-\text { narrow }} \Delta \nu=400, N^{\prime}=\tau_{\text {min }} / \tau_{\mu-\text { narrow }}=15$, and $\varsigma \sim 2.1$; given these parameters, a simple simulation (described in Appendix E) shows that the bias to the degree of polarization is less than 4\%. Although the subpulse structure of pulsars does not consist of a sequence of rectangular impulses, this tractable basic model is sufficient to demonstrate to first order that amplitude modulation by narrow subpulses with log-normally distributed amplitudes does not significantly reduce the statistical degrees of freedom in the pulsar signal. Therefore, as neither wave coherence nor amplitude modulation reproduce the observed correlation between degree of polarization and temporal resolution, it is unlikely that the correlation can be dismissed as a consequence of the bias associated with small number statistics.

\section{B. COMPARISON WITH CARDOSO (1991)}

Equations (15) through (21) incorporate a couple of conventions that are different to those used by Cardoso (1991). Although these differences are of no consequence in this work, they deserve mention. First, note that Equation (6) of Cardoso (1991) includes the Hermitian transpose of the second operand B in the definitions of the tensor products, $\otimes$ and $\tilde{\otimes}$. To maintain consistency with the notation of Equation (8), where the tensor product and adjoint operator are separately and explicitly invoked, the implicit adjoint operator is omitted from the definitions in Equations (15) and (16) and therefore does not appear on the right hand sides of Equations (24) and (25). This distinction is inconsequential because in this work $\boldsymbol{\rho}, \mathbf{A}$ and $\mathbf{B}$ are self-adjoint and linearly related to real-valued Stokes parameters.

Also note that the double contraction defined in Equation (21) uses an index notation convention that is different to the one that appears on the left hand sides of Equation (6) in Cardoso (1991). As inferred from the indeces on the tensor coordinates in the text that follows Equation (6), the convention used by Cardoso (1991) is defined by transposing $\boldsymbol{\rho}$ in Equation (21). Again, this difference is of no consequence because the tensor coordinates are of secondary importance to the transformation properties of $\mathbf{A} \otimes \mathbf{B}$ and $\mathbf{A} \tilde{\otimes} \mathbf{B}$.

More important than these minor differences is the fundamental consistency between the findings of Cardoso (1991) and the results of Section 2. After accounting for the difference in index notation convention mentioned above, the third symmetry identified by Cardoso (1991) is easily seen to be equivalent to the transpose over contravariant tensor indeces that converts $\mathbf{A} \otimes \mathbf{B}$ into $\mathbf{A} \tilde{\otimes} \mathbf{B}$ (and vice versa). This leads to a useful interpretation of the "Rank One Lemma" demonstrated by Cardoso (1991). In the special case of $100 \%$ polarized radiation, the coherency matrix has a rank of one because it has only one non-zero eigenvalue (e.g. Eq. [7] of van Straten 2009, and the discussion that follows). In this case, the Lorentz invariant of the associated Stokes parameters $S^{2}=0$ and $S \otimes S=S \tilde{\otimes} S$; that is, in the case of $100 \%$ polarization, the two tensor products are invariant under the third symmetry and equal to each other. Note that the instantaneous coherency matrix is always rank one and the instantaneous Stokes parameters always satisfy $s \otimes s=s \tilde{\otimes} s$.

\section{DERIVATION OF EQUATION (30)}

To derive Equation (30), substitute $\mathbf{A}=A_{\alpha} \boldsymbol{\sigma}_{\alpha} / 2$ and $\mathbf{B}=B_{\beta} \boldsymbol{\sigma}_{\beta} / 2$ into Equation (27) to express it in terms of the associated Stokes parameters,

$$
\left\{\mathbf{M}_{\tilde{\otimes}}(A, B)\right\}_{\mu}^{\nu}=\frac{1}{2} \boldsymbol{\sigma}_{\mu}:\left(\mathbf{A} \boldsymbol{\sigma}_{\nu} \mathbf{B}\right)=\frac{1}{8} A_{\alpha} B_{\beta} \operatorname{Tr}\left[\boldsymbol{\sigma}_{\mu} \boldsymbol{\sigma}_{\alpha} \boldsymbol{\sigma}_{\nu} \boldsymbol{\sigma}_{\beta}\right]
$$

For each of the 16 elements of $\mathbf{M}_{\tilde{\otimes}}(A, B)$, a total of 16 terms arise in the above double sum over $\alpha$ and $\beta$. The trace of a product of four Pauli matrices has been derived in Appendix B of Melrose (1993a). To arrive at a more compact, coordinate-free representation, the $4 \times 4$ matrix is partitioned into the following four parts.

$$
\begin{aligned}
& \text { 1. } \nu=\mu=0 \\
& \text { 2. } \nu=0 \text { and } \mu=j>0 \\
& \text { 3. } \nu=k>0 \text { and } \mu=0
\end{aligned}
$$


4. $\nu=k>0$ and $\mu=j>0$

For each part, the double sum is solved as follows.

1. When $\mu=\nu=0$, the result is simply one quarter of the Euclidean inner product of the Stokes four-vectors; i.e.,

$$
\left\{\mathbf{M}_{\tilde{\otimes}}(A, B)\right\}_{0}^{0}=\frac{1}{2} \operatorname{Tr}[\mathbf{A B}]=\frac{1}{4} A_{\mu} B_{\mu} .
$$

2. When $\mu=j>0$ and $\nu=0$, the double sum can be partitioned into the same four parts

(a) $\alpha=\beta=0: \operatorname{Tr}\left[\boldsymbol{\sigma}_{j}\right]=0$

(b) $\alpha=0$ and $\beta=b>0: \operatorname{Tr}\left[\boldsymbol{\sigma}_{j} \boldsymbol{\sigma}_{b}\right]=2 \delta_{j b}$

(c) $\alpha=a>0$ and $\beta=0: \operatorname{Tr}\left[\boldsymbol{\sigma}_{j} \boldsymbol{\sigma}_{a}\right]=2 \delta_{j a}$

(d) $\alpha=a>0$ and $\beta=b>0: \operatorname{Tr}\left[\boldsymbol{\sigma}_{j} \boldsymbol{\sigma}_{a} \boldsymbol{\sigma}_{b}\right]=2 i \epsilon_{j a b}$

where $\epsilon_{j k l}$ is the rank 3 permutation pseudotensor, yielding

$$
\left\{\mathbf{M}_{\tilde{\otimes}}(A, B)\right\}_{j}^{0}=\frac{1}{4}\left(A_{0} B_{j}+A_{j} B_{0}+i \epsilon_{j a b} A_{a} B_{b}\right) .
$$

3. Similarly, when $\mu=0$ and $\nu=k>0$,

$$
\left\{\mathbf{M}_{\tilde{\otimes}}(A, B)\right\}_{0}^{k}=\frac{1}{4}\left(A_{k} B_{0}+A_{0} B_{k}+i \epsilon_{a k b} A_{a} B_{b}\right)
$$

4. When $\mu=j>0$ and $\nu=k>0$, partition the double sum to yield

(a) $\alpha=\beta=0: \operatorname{Tr}\left[\boldsymbol{\sigma}_{j} \boldsymbol{\sigma}_{k}\right]=2 \delta_{k j}$

(b) $\alpha=0$ and $\beta=b>0: \operatorname{Tr}\left[\boldsymbol{\sigma}_{j} \boldsymbol{\sigma}_{k} \boldsymbol{\sigma}_{b}\right]=2 i \epsilon_{j k b}$

(c) $\alpha=a>0$ and $\beta=0: \operatorname{Tr}\left[\boldsymbol{\sigma}_{j} \boldsymbol{\sigma}_{a} \boldsymbol{\sigma}_{k}\right]=2 i \epsilon_{j a k}$

(d) $\alpha=a>0$ and $\beta=b>0: \operatorname{Tr}\left[\boldsymbol{\sigma}_{j} \boldsymbol{\sigma}_{a} \boldsymbol{\sigma}_{k} \boldsymbol{\sigma}_{b}\right]=2\left(\delta_{j a} \delta_{k b}-\delta_{j k} \delta_{a b}+\delta_{j b} \delta_{a k}\right)$

and

$$
\left\{\mathbf{M}_{\tilde{\otimes}}(A, B)\right\}_{j}^{k}=\frac{1}{4}\left(A_{j} B_{k}+A_{k} B_{j}+\delta_{k j} A \cdot B+i \epsilon_{j k b} A_{0} B_{b}+i \epsilon_{j a k} A_{a} B_{0}\right) .
$$

Noting that $A_{\mu} B_{\mu}=2 A_{0} B_{0}-A \cdot B$, Equations (51) through (54) can be combined to produce

$$
\mathbf{M}_{\tilde{\otimes}}(A, B)=\frac{1}{4}(A \otimes B+B \otimes A-\boldsymbol{\eta} A \cdot B+i A \wedge B) .
$$

Here, $A \wedge B$ is the covariant exterior product of $A$ and $B$, a $4 \times 4$ antisymmetric matrix (i.e. $A \wedge B=-B \wedge A$ ) defined by

$$
\{A \wedge B\}_{\mu}^{\nu} \equiv \epsilon_{\mu \alpha \nu \beta} A^{\alpha} B^{\beta},
$$

where $\epsilon_{\alpha \beta \delta \gamma}$ is the rank 4 permutation pseudotensor, $A^{\alpha}=\eta^{\alpha \gamma} A_{\gamma}$ and $B^{\beta}=\eta^{\beta \gamma} B_{\gamma}$. Finally, as in Equation (28), define $A \tilde{\otimes} B=2 \mathbf{M}_{\tilde{\otimes}}(A, B)$.

\section{COMPARISON WITH CORDES ET AL. (1978)}

To distinguish between disjoint and superposed modes, Section III. c) of CRB proposes a statistical test that starts by describing the linear polarization $L=Q+i U$ at a given pulse longitude as a linear combination of orthogonally polarized modes, $L=L_{1}+L_{2}=\left(\left|L_{1}\right|-\left|L_{2}\right|\right) \exp (2 i \psi)$. It is asserted that intermediate position angles between $\psi$ and $\psi+\pi / 2$ do not occur; therefore, without any loss of generality, choose $\psi=0$ and define the orthogonally polarized modes such that the instantaneous Stokes parameters $a=2\left[I_{1},\left|L_{1}\right|, 0,0\right]$ and $b=2\left[I_{2},-\left|L_{2}\right|, 0,0\right]$. Note that, because $a_{2}=a_{3}=b_{2}=b_{3}=0$, the standard deviations $\varsigma_{2}=\varsigma_{3}=0$ and, referring to Equation (45), this implies that the Lorentz invariant $S^{2}=0$. That is, the modes defined in Section III. c) of CRB are implicitly assumed to be $100 \%$ polarized. In this case, the mode intensities, $I_{1}=\left|L_{1}\right|$ and $I_{2}=\left|L_{2}\right|$; however, to facilitate comparison with CRB, $I_{1}$, $I_{2},\left|L_{1}\right|$, and $\left|L_{2}\right|$ are treated as distinct random variates until these two equalities are required.

CRB also assume that both modes occur equally frequently such that, when the modes are disjoint, the population mean Stokes parameters are $S=(A+B) / 2=[\langle I\rangle,\langle L\rangle, 0,0]$, where $A=\langle a\rangle$ and $B=\langle b\rangle$. After substitution of the 
above definitions and $n=1$ and $F=0.5$ into Equation (39), rearrange and solve for the moments of the instantaneous Stokes parameters,

$$
\begin{aligned}
\left\langle s_{\mu} s_{\nu}\right\rangle=\left\{\mathbf{C}_{d}\right\}_{\mu}^{\nu}+\left\langle s_{\mu}\right\rangle\left\langle s_{\nu}\right\rangle & =\frac{1}{2}\left(\left\{\mathbf{C}_{A}\right\}_{\mu}^{\nu}+\left\{\mathbf{C}_{B}\right\}_{\mu}^{\nu}\right)+\frac{1}{4}\left[\left(A_{\mu}-B_{\mu}\right)\left(A_{\nu}-B_{\nu}\right)+\left(A_{\mu}+B_{\mu}\right)\left(A_{\nu}+B_{\nu}\right)\right] \\
& =\frac{1}{2}\left(\left\langle a_{\mu} a_{\nu}\right\rangle+\left\langle b_{\mu} b_{\nu}\right\rangle\right) .
\end{aligned}
$$

The above equation yields $\left\langle L^{2}\right\rangle=\left\langle s_{1}^{2}\right\rangle=2\left(\left\langle L_{1}^{2}\right\rangle+\left\langle L_{2}^{2}\right\rangle\right)$ and, noting that $\left\langle I_{1} I_{2}\right\rangle=0$ for mutually exclusive modes, $\left\langle I^{2}\right\rangle=\left\langle s_{0}^{2}\right\rangle=2\left\langle\left(I_{1}+I_{2}\right)^{2}\right\rangle$. Therefore, the covariances between the instantaneous Stokes parameters of disjoint modes described in Section 3.1 are consistent with disjoint modes in Equation (5) of CRB. Furthermore, although not explicitly noted in CRB, because the modes are implicitly assumed to be $100 \%$ polarized, $\left\langle d_{L}^{2}\right\rangle \equiv\left\langle L^{2}\right\rangle /\left\langle I^{2}\right\rangle=1$ in the case of disjoint modes.

For superposed modes, divide $a$ and $b$ by 2 , then substitute $n=1$ and $S=A+B$ into Equation (42) and solve for the moments,

$$
\begin{aligned}
\left\langle s_{\mu} s_{\nu}\right\rangle=\left\{\mathbf{C}_{s}\right\}_{\mu}^{\nu}+\left\langle s_{\mu}\right\rangle\left\langle s_{\nu}\right\rangle & =\left\{\mathbf{C}_{A}\right\}_{\mu}^{\nu}+\left\{\mathbf{C}_{B}\right\}_{\mu}^{\nu}+\{A \tilde{\odot} B\}_{\mu}^{\nu} \\
& =\left\langle a_{\mu} a_{\nu}\right\rangle+\left\langle b_{\mu} b_{\nu}\right\rangle+2 A_{\mu} B_{\nu}+2 A_{\nu} B_{\mu}-\eta_{\mu}^{\nu} A \cdot B .
\end{aligned}
$$

Owing to the assumption that the modes are $100 \%$ polarized, $A \cdot B=\left\langle I_{1}\right\rangle\left\langle I_{2}\right\rangle+\left\langle\left|L_{1}\right|\right\rangle\left\langle\left|L_{2}\right|\right\rangle=2\left\langle\left|L_{1}\right|\right\rangle\left\langle\left|L_{2}\right|\right\rangle$, and the above equation yields $\left\langle L^{2}\right\rangle=\left\langle s_{1}^{2}\right\rangle=\left\langle L_{1}^{2}\right\rangle+\left\langle L_{2}^{2}\right\rangle-2\left\langle\left|L_{1}\right|\right\rangle\left\langle\left|L_{2}\right|\right\rangle$. Furthermore, because the mode intensities are uncorrelated, $\left\langle I_{1} I_{2}\right\rangle=\left\langle I_{1}\right\rangle\left\langle I_{2}\right\rangle$ and $\left\langle I^{2}\right\rangle=\left\langle s_{0}^{2}\right\rangle=\left\langle\left(I_{1}+I_{2}\right)^{2}\right\rangle$. Therefore, the covariances between the instantaneous Stokes parameters of superposed modes as described in Section 3.2 are consistent with superposition of modes in Equation (5) of CRB only if the modes are assumed to be $100 \%$ polarized.

To arrive at the above results for disjoint and superposed modes, it is necessary to consider the instantaneous Stokes parameters; that is, to be consistent with CRB, the Stokes sample size must be unity $(n=1)$. Under this assumption, mutually exclusive modes are disjoint by definition. To consider a Stokes sample composed of mutually exclusive and orthogonally polarized instances, replace the instantaneous Stokes parameters $a$ and $b$ with the subsample mean Stokes parameters $\bar{A}^{\prime}=2\left[I_{1},\left|L_{1}\right|, 0,0\right]$ and $\bar{B}^{\prime}=2\left[I_{2},-\left|L_{2}\right|, 0,0\right]$, formed after respectively averaging over all instances in mode $A$ and all instances in mode $B$. In this case, the matrix of covariances between the sample mean Stokes parameters is given by

$$
\overline{\mathbf{C}}_{c}=f^{2} \overline{\mathbf{C}}_{A}^{\prime}+(1-f)^{2} \overline{\mathbf{C}}_{B}^{\prime}
$$

where $\overline{\mathbf{C}}_{A}^{\prime}=\mathbf{C}_{A}(f n)^{-1}$ and $\overline{\mathbf{C}}_{B}^{\prime}=\mathbf{C}_{B}[(1-f) n]^{-1}$ are the covariances between the sub-sample mean Stokes parameters after averaging over $f n$ instances in mode $A$ and $(1-f) n$ instances in mode $B$, respectively. Substitute $f=0.5$ into the above equation and rearrange to yield the moments of the composite sample mean Stokes parameters,

$$
\begin{aligned}
\left\langle\bar{S}_{\mu} \bar{S}_{\nu}\right\rangle=\left\{\overline{\mathbf{C}}_{c}\right\}_{\mu}^{\nu}+\left\langle\bar{S}_{\mu}\right\rangle\left\langle\bar{S}_{\nu}\right\rangle & =\frac{1}{4}\left(\left\{\overline{\mathbf{C}}_{A}^{\prime}\right\}_{\mu}^{\nu}+\left\{\overline{\mathbf{C}}_{B}^{\prime}\right\}_{\mu}^{\nu}\right)+\frac{1}{4}\left(A_{\mu}+B_{\mu}\right)\left(A_{\nu}+B_{\nu}\right) \\
& =\frac{1}{4}\left(\left\langle\bar{A}_{\mu}^{\prime} \bar{A}_{\nu}^{\prime}\right\rangle+\left\langle\bar{B}_{\mu}^{\prime} \bar{B}_{\nu}^{\prime}\right\rangle+A_{\mu} B_{\nu}+A_{\nu} B_{\mu}\right) .
\end{aligned}
$$

The above equation yields $\left\langle L^{2}\right\rangle=\left\langle\bar{S}_{1}^{2}\right\rangle=\left\langle L_{1}^{2}\right\rangle+\left\langle L_{2}^{2}\right\rangle-2\left\langle\left|L_{1}\right|\right\rangle\left\langle\left|L_{2}\right|\right\rangle$ and $\left\langle I^{2}\right\rangle=\left\langle\bar{S}_{0}^{2}\right\rangle=\left\langle\left(I_{1}+I_{2}\right)^{2}\right\rangle$. Therefore, the second moments of the composite sample mean Stokes parameters are also consistent with the superposition of modes in Equation (5) of CRB. That is, given only the second moments of the sample mean total and linearly polarized intensities, it is not possible to distinguish between superposed modes and Stokes samples that are composed of mutually exclusive states.

\section{E. VERIFICATION BY SIMULATION}

To verify the equations presented in this paper, the following Monte Carlo simulation was repeatedly performed over a wide range of input parameters and conditions.

1. Generate a sequence of $M$ random electric field vector instances $\boldsymbol{e}$, each with statistically independent and identically distributed (iid) circular complex normal components; such a sequence is described by the population mean Stokes parameters $[1,0,0,0]$.

2. To yield the desired population mean Stokes parameters, $S_{\mu}$, transform each electric field vector instance by the Hermitian square root of $2 \rho=S_{\mu} \sigma_{\mu}$. 
3. Optionally perform amplitude modulation by multiplying each instance of $e$ by an iid random variate $u$ that is drawn from a log-normal distribution. The log-normally distributed variate is generated from a normally distributed iid variate with zero mean and standard deviation $\varsigma$ and is normalized by the mean of the distribution, $\langle u\rangle=\exp \left(\varsigma^{2} / 2\right)$, such that the mean of the amplitude modulating function is unity. To simulate rectangular subpulses defined by the sub-sample size $n$, as described in Appendix A, a single value of $u$ is applied to $n^{\prime}$ consecutive instances of $\boldsymbol{e}$.

4. If simulating superposed samples, repeat all of the previous steps to produce $M$ instances of the electric field vector in the other mode then, for each instance of the electric field vectors from modes $A$ and $B$, produce $M$ new instances $\boldsymbol{e}=\boldsymbol{e}_{A}+\boldsymbol{e}_{B}$.

5. Compute the instantaneous Stokes parameters, $s_{\mu}=e^{\dagger} \sigma_{\mu} \boldsymbol{e}$.

6. Optionally divide the sequence of $M$ instantaneous Stokes vectors into mutually exclusive Stokes samples of $n$ instances, yielding a sequence of $N=M / n$ Stokes samples. This step is not optional when simulating composite samples.

7. If simulating composite samples, replace $(1-f) n$ instances in each Stokes sample with instantaneous Stokes vectors in the other mode.

8. If simulating disjoint samples, replace $(1-F) N$ Stokes samples with Stokes samples that contain only instantaneous Stokes vectors in the other mode.

9. For each Stokes sample, compute the sample mean Stokes parameters $\bar{S}_{\mu}$.

10. Compute the $4 \times 4$ covariances between the Stokes parameters using either the $M$ instantaneous Stokes parameters or the $N$ sample mean Stokes parameters. Verify that the computed covariance matrix matches the theoretical prediction within the uncertainty due to noise.

The above simulation is implemented in $\mathrm{C}++$ and is freely available as the EPSIC open source software package for simulating the polarization of electromagnetic radiation ${ }^{7}$.

\section{REFERENCES}

Allen, M., \& Melrose, D. 1982, PASA, 4, 365

Amblard, P., Gaeta, M., \& Lacoume, J. 1996, Signal Processing, 53,1

Backer, D. C. 1970, Nature, 228, 42

Backer, D. C., \& Rankin, J. M. 1980, ApJS, 42, 143

Barakat, R. 1963, J. Opt. Soc. Am., 53, 317

Born, M., \& Wolf, E. 1970, Principles of Optics: Electromagnetic Theory of Propagation, Interference and Diffraction of Light (New York: Pergamon)

Bracewell, R. 1986, The Fourier Transform and its Applications (New York: McGraw-Hill)

Britton, M. C. 2000, ApJ, 532, 1240

Brosseau, C., \& Barakat, R. 1992, Opt. Comm., 91, 408

Burns, W. R., \& Clark, B. G. 1969, A\&A, 2, 280

Cairns, I. H., Johnston, S., \& Das, P. 2003, MNRAS, 343, 512

Cardoso, J.-F. 1991, in ICASSP 91. 1991 International

Conference on Acoustics, Speech, and Signal Processing (New

York: IEEE), 3109 - 3112

Cloude, S. 1986, Optik, 75, 26

Cognard, I., Shrauner, J. A., Taylor, J. H., \& Thorsett, S. E. 1996, ApJ, 457, L81

Cordes, J. M. 1976, ApJ, 208, 944

Cordes, J. M. 1986, ApJ, 311, 183

7 See http://straten.github.io/epsic
Cordes, J. M., Bhat, N. D. R., Hankins, T. H., McLaughlin, M. A., \& Kern, J. 2004, ApJ, 612, 375

Cordes, J. M., \& Hankins, T. H. 1977, ApJ, 218, 484

Cordes, J. M., Rankin, J. M., \& Backer, D. C. 1978, ApJ, 223, 961

Cordes, J. M., Rickett, B. J., Stinebring, D. R., \& Coles, W. A. 2006, ApJ, 637, 346

Edwards, R. T. 2004, A\&A, 426, 677

Edwards, R. T., \& Stappers, B. W. 2002, A\&A, 393, 733

-. 2003, A\&A, 407, 273

-. 2004, A\&A, 421, 681

Ekers, R. D., \& Moffet, A. T. 1969, ApJ, 158, L1

Eriksson, J., Ollila, E., \& Koivunen, V. 2010, IEEE Transactions on Signal Processing, 58, 5400

Gangadhara, R. 1997, A\&A, 327, 155

Gangadhara, R. T., Xilouris, K. M., von Hoensbroech, A., et al. 1999, A\&A, 342, 474

Gil, J. A., Snakowski, J. K., \& Stinebring, D. R. 1991, A\&A, 242, 119

Goodman, L. A. 1960, Journal of the American Statistical Association, 55, 708

Goodman, N. R. 1963, Ann. of Math. Stat., 34, 152

Hankins, T. H., Kern, J. S., Weatherall, J. C., \& Eilek, J. A. 2003, Nature, 422, 141

Harding, A. K., \& Tademaru, E. 1981, ApJ, 243, 597

Heiles, C., Campbell, D. B., \& Rankin, J. M. 1970, Nature, 226, 529

Jenet, F. A., \& Gil, J. 2003, ApJ, 596, L215 
Karastergiou, A., Johnston, S., \& Kramer, M. 2003, A\&A, 404, 325

Karastergiou, A., Roberts, S. J., Johnston, S., et al. 2011, MNRAS, 415, 251

Kendall, M. G., Stuart, A., \& Ord, J. K. 1987, Kendall's Advanced Theory of Statistics (London: Charles Griffin)

Kollo, T., \& von Rosen, D. 2005, Advanced Multivariate Statistics with Matrices (Dordrecht: Springer)

Krishnamohan, S., \& Downs, G. S. 1983, ApJ, 265, 372

Liu, K., Karuppusamy, R., Lee, K. J., et al. 2015, MNRAS, 449, 1158

Manchester, R. N., Taylor, J. H., \& Huguenin, G. R. 1975, ApJ, 196, 83

McCullagh, P. 1987, Tensor Methods in Statistics, Monographs on Statistics and Applied Probability (London: Chapman and Hall)

McKinnon, M., \& Stinebring, D. 1998, ApJ, 502, 883

McKinnon, M. M. 2002, ApJ, 568, 302

-. 2003a, ApJ, 590, 1026

-. 2003b, ApJS, 148, 519

—. 2004, ApJ, 606, 1154

—. 2006, ApJ, 645, 551

McKinnon, M. M., \& Stinebring, D. R. 2000, ApJ, 529, 435

Melrose, D. B. 1993a, Journal of Plasma Physics, 50, 267

-. 1993b, Journal of Plasma Physics, 50, 283

Melrose, D. B., \& Macquart, J.-P. 1998, ApJ, 505, 921

Mendel, J. 1991, Proc. IEEE, 79, 278
Osłowski, S., van Straten, W., Bailes, M., Jameson, A., \& Hobbs, G. 2014, MNRAS, 441, 3148

Osłowski, S., van Straten, W., Demorest, P., \& Bailes, M. 2013, MNRAS, 430, 416

Picinbono, B. 1994, Trans. Sig. Proc., 42, 3473

Popov, M. V., Bartel, N., Cannon, W. H., et al. 2002, Astronomy Reports, 46, 206

Ransom, S. M., Eikenberry, S. S., \& Middleditch, J. 2002, AJ, 124,1788

Rickett, B. J. 1975, ApJ, 197, 185

Smirnov, O. M. 2011, A\&A, 531, A159

Stinebring, D. R., Cordes, J. M., Rankin, J. M., Weisberg, J. M., \& Boriakoff, V. 1984, ApJS, 55, 247

Stinebring, D. R., McLaughlin, M. A., Cordes, J. M., et al. 2001, ApJ, 549, L97

Sultan, S. A., \& Tracy, D. S. 1996, Linear Algebra and its Applications, 237/238, 191

Taylor, J. H., Huguenin, G. R., Hirsch, R. M., \& Manchester, R. N. 1971, Astrophys. Lett., 9, 205

Taylor, J. H., Manchester, R. N., \& Huguenin, G. R. 1975, ApJ, 195,513

van Straten, W. 2009, ApJ, 694, 1413

—. 2010, ApJ, 719, 985

Walker, M. A., Melrose, D. B., Stinebring, D. R., \& Zhang, C. M. 2004, MNRAS, 354, 43

Weltevrede, P., Edwards, R. T., \& Stappers, B. W. 2006, A\&A, 445,243

Weltevrede, P., Stappers, B. W., \& Edwards, R. T. 2007, A\&A, 469,607

\section{APPENDIX}

The following additional material was not submitted to The Astrophysical Journal and was not reviewed by the referee. It is provided as further information for the interested reader.

\section{F.1. Error in Section 5.2 of Amblard, Gaeta, and Lacoume (1996)}

In Statistics for complex variables and signals - Part I: Variables, Amblard, Gaeta, and Lacoume (1996) study the higher-order statistics of multivariate complex random values using tensor notation. At the bottom of the left-hand column of page 9, they assert that the fourth-order cumulant $C_{2}^{2}$ of the vector $\boldsymbol{Z}$ is related to the fourth-order moment $M_{2}^{2}$ and second-order moment $M_{1}^{1}$ as follows

$$
C_{2}^{2}=M_{2}^{2}-2 M_{1}^{1} \otimes M_{1}^{1}
$$

In the special case that the vector $\boldsymbol{Z}$ has a complex-valued normal distribution, then the fourth-order cumulant $C_{2}^{2}$ is equal to zero, such that

$$
M_{2}^{2}=2 M_{1}^{1} \otimes M_{1}^{1}
$$

Now, if the components of $\boldsymbol{Z}$ are uncorrelated and have unit variance, then the second moment $M_{1}^{1}$ is the identity matrix; i.e.

$$
\left\{M_{1}^{1}\right\}_{i}^{j}=\delta_{i}^{j}
$$

where $\delta_{i}^{j}$ is the Kronecker delta. Using index notation, the fourth moment is defined as

$$
\left\{M_{2}^{2}\right\}_{j n}^{i m}=\left\langle Z_{i} Z_{m} Z_{j}^{*} Z_{n}^{*}\right\rangle
$$

However, Equation (63) above says

$$
\left\{M_{2}^{2}\right\}_{j n}^{i m}=2 M_{1}^{1} \otimes M_{1 j n}^{1 i m}=2 \delta_{j}^{i} \delta_{n}^{m},
$$

which breaks down when $m=j=a$ and $n=i=b$ and $a \neq b$, such that

$$
\left\{M_{2}^{2}\right\}_{b a}^{a b}=\left\langle Z_{a} Z_{b} Z_{b}^{*} Z_{a}^{*}\right\rangle=\left\langle\left|Z_{a}\right|^{2}\left|Z_{b}\right|^{2}\right\rangle
$$


where $w w^{*}=|w|^{2}$ and $a$ and $b$ are constants; i.e. no summation over indeces is implied. Now $\left|Z_{a}\right|^{2} \geq 0$ and $\left|Z_{b}\right|^{2} \geq 0$; therefore, the expectation of their product is greater than zero. However, Equation (63) dictates that this fourth moment must be zero; i.e.

$$
\left\{M_{2}^{2}\right\}_{b a}^{a b}=2 \delta_{b}^{a} \delta_{a}^{b}=0
$$

\section{F.2. Double contraction of Cardoso (1991)}

In Super-symmetric decomposition of the fourth-order cumulant tensor. Blind identification of more sources than sensors., Cardoso (1991) studies the higher-order statistics of multivariate complex random values using tensor notation. Equation (6) of Cardoso (1991) defines two tensor products in terms of their transformation properties. The left hand sides of these equations include a double contraction, the definition of which can be inferred from the coordinates of the tensors provided in the text that follows, namely

$$
\begin{aligned}
& \left\{A \otimes_{1} B\right\}_{i j k l} \equiv a_{i j} b_{k l}^{*} \\
& \left\{A \otimes_{2} B\right\}_{i j k l} \equiv a_{i k} b_{j l}^{*} .
\end{aligned}
$$

To arrive at the above equations, the double contraction must be defined as

$$
\{U M\}_{i j} \equiv u_{i j k l} m_{k l} \text {. }
$$

Note that, in the above equation, no distinction is made between covariant and contravariant indeces; that is, contractions are performed over pairs of similar indeces. To show that the above definition is necessary, start with the definitions of the outer products,

$$
\begin{aligned}
\left(A \otimes_{1} B\right) M & \equiv A \operatorname{Tr}\left[M B^{\mathrm{H}}\right] \\
\left(A \otimes_{2} B\right) M & \equiv A M B^{\mathrm{H}} .
\end{aligned}
$$

In index notation, the left hand side of Equation (70) is

$$
\left\{\left(A \otimes_{1} B\right) M\right\}_{i j} \equiv\left\{A \otimes_{1} B\right\}_{i j k l} m_{k l}=a_{i j} b_{k l}^{*} m_{k l}
$$

and the right hand side is

$$
\left\{A \operatorname{Tr}\left[M B^{\mathrm{H}}\right]\right\}_{i j}=a_{i j}\left\{M B^{\mathrm{H}}\right\}_{k k}=a_{i j} b_{k l}^{*} m_{k l}
$$

Similarly, the left hand side of Equation (71) is

$$
\left\{\left(A \otimes_{2} B\right) M\right\}_{i j} \equiv\left\{A \otimes_{2} B\right\}_{i j k l} m_{k l}=a_{i k} b_{j l}^{*} m_{k l}
$$

and the right hand side is

$$
\left\{A M B^{\mathrm{H}}\right\}_{i j}=a_{i k}\left\{M B^{\mathrm{H}}\right\}_{k j}=a_{i k} b_{j l}^{*} m_{k l}
$$

\section{F.3. Equations (15) and (16) are consistent with Equations (24) and (25), respectively}

Substitute the left hand side of Equation (24) into the left hand side of Equation (21) and expand the right hand side of Equation (21) using the right hand side of Equation (15) to arrive at

$$
\{(\mathbf{A} \otimes \mathbf{B}): \boldsymbol{\rho}\}_{i}^{j}=A_{i}^{j} B_{k}^{l} \rho_{l}^{k} .
$$

Now expand the right hand side of Equation (24) using index notation to arrive at the same result

$$
\{\mathbf{A} \operatorname{Tr}[\boldsymbol{\rho} \mathbf{B}]\}_{i}^{j}=A_{i}^{j}\{\boldsymbol{\rho} \mathbf{B}\}_{l}^{l}=A_{i}^{j} B_{k}^{l} \rho_{l}^{k}
$$

Similarly, substitute the left hand side of Equation (25) into the left hand side of Equation (21) and expand the right hand side of Equation (21) using the right hand side of Equation (16) to arrive at

$$
\{(\mathbf{A} \tilde{\otimes} \mathbf{B}): \boldsymbol{\rho}\}_{i}^{j}=A_{i}^{l} B_{k}^{j} \rho_{l}^{k} .
$$

Now expand the right hand side of Equation (25) using index notation to arrive at the same result

$$
\{\mathbf{A} \rho \mathbf{B}\}_{i}^{j}=A_{i}^{l}\{\boldsymbol{\rho} \mathbf{B}\}_{l}^{j}=A_{i}^{l} B_{k}^{j} \rho_{l}^{k}
$$




\section{F.4. Verification of Equation (23)}

Equation (23) can be verified by substituting it into the right hand side of Equation (22), yielding

$$
\mathrm{RHS}=\frac{1}{2} \boldsymbol{\sigma}_{\mu}:\left(\frac{1}{2} M_{\kappa}^{\lambda} \boldsymbol{\sigma}_{\kappa} \otimes \boldsymbol{\sigma}_{\lambda}\right): \boldsymbol{\sigma}_{\nu}=\frac{1}{4} M_{\kappa}^{\lambda} \boldsymbol{\sigma}_{\mu}:\left(\boldsymbol{\sigma}_{\kappa} \otimes \boldsymbol{\sigma}_{\lambda}\right): \boldsymbol{\sigma}_{\nu}
$$

The above expression can be reorganized using Equation (24) to yield

$$
\mathrm{RHS}=\frac{1}{4} M_{\kappa}^{\lambda}\left(\sigma_{\mu}: \sigma_{\kappa}\right)\left(\sigma_{\lambda}: \sigma_{\nu}\right)
$$

Then, use

$$
\sigma_{\mu}: \sigma_{\nu}=\operatorname{Tr}\left[\sigma_{\mu} \sigma_{\nu}\right]=2 \delta_{\mu \nu} .
$$

to arrive at the left hand side of Equation (22) - done!

$$
\mathrm{RHS}=M_{\kappa}^{\lambda} \delta_{\mu \kappa} \delta_{\nu \lambda}=M_{\mu}^{\nu}=\mathrm{LHS} .
$$

\section{F.5. Derivation of Equation (32)}

Starting with $\mathbf{U}=\boldsymbol{\kappa}_{2 ; 2}(\boldsymbol{e})$, substitute Equation (13) into Equation (22), multiply by two, and apply Equations (26) and (27) to arrive at

$$
\begin{aligned}
Q_{\mu}^{\nu} & =2 M_{\mu}^{\nu}=\boldsymbol{\sigma}_{\mu}: \boldsymbol{\kappa}_{2 ; 2}(\boldsymbol{e}): \boldsymbol{\sigma}_{\nu} \\
& =\boldsymbol{\sigma}_{\mu}:(\langle\boldsymbol{r} \otimes \boldsymbol{r}\rangle-\boldsymbol{\rho} \otimes \boldsymbol{\rho}-\boldsymbol{\rho} \tilde{\otimes} \boldsymbol{\rho}): \boldsymbol{\sigma}_{\nu} \\
& =\left\langle 2\left\{\mathbf{M}_{\otimes}(\boldsymbol{r}, \boldsymbol{r})\right\}_{\mu}^{\nu}\right\rangle-2\left\{\mathbf{M}_{\otimes}(\boldsymbol{\rho}, \boldsymbol{\rho})\right\}_{\mu}^{\nu}-2\left\{\mathbf{M}_{\tilde{\otimes}}(\boldsymbol{\rho}, \boldsymbol{\rho})\right\}_{\mu}^{\nu} .
\end{aligned}
$$

Then apply Equations (28) and (30) to express the above equation in terms of the instantaneous and population mean Stokes parameters, $s$ and $S$, associated with $\boldsymbol{r}$ and $\boldsymbol{\rho}$, respectively,

$$
\mathbf{Q}=\left\langle s \otimes s^{T}\right\rangle-S \otimes S^{T}-S \tilde{\otimes} S=\mathbf{C}-S \tilde{\otimes} S
$$

where the last equality follows from Equation (33) and the definition of $\mathbf{C}$ following Equation (32).

F.6. Noise bias is consistent with Equation (9) of Cordes 83 Hankins (1977)

Assuming that the noise is normally distributed and unpolarized, the last two terms of Equation (47) are

$$
\mathbf{B}=\mathbf{C}_{\mathrm{N}}+S_{\mathrm{S}} \tilde{\odot} S_{\mathrm{N}}=S_{\mathrm{N}} \tilde{\otimes} S_{\mathrm{N}}+S_{\mathrm{S}} \tilde{\odot} S_{\mathrm{N}}
$$

and the biases to the variances of the Stokes parameters are given by

$$
b_{\mu}=B_{\mu \mu}=S_{\mathrm{N}, \mu}\left(S_{\mathrm{N}, \mu}+2 S_{\mathrm{S}, \mu}\right)-\frac{1}{2} \eta_{\mu \mu} S_{\mathrm{N}, 0}\left(S_{\mathrm{N}, 0}+2 S_{\mathrm{S}, 0}\right)
$$

Noting that $S_{\mathrm{N}, \mathrm{j}}=0$ for $j>0$, the biases to the variances of all four Stokes parameters are equal to

$$
b_{\mu}=\frac{1}{2} S_{\mathrm{N}, 0}\left(S_{\mathrm{N}, 0}+2 S_{\mathrm{S}, 0}\right)
$$

When comparing the above bias estimate with Equation (9) of Cordes \& Hankins (1977), note that $S_{\mathrm{S}, 0}=\left\langle I_{r_{o n}}\right\rangle+\left\langle I_{l_{o n}}\right\rangle$ and, for unpolarized system and sky noise, $\left\langle I_{r_{o f f}}\right\rangle=\left\langle I_{l_{o f f}}\right\rangle=S_{\mathrm{N}, 0} / 2$. The biases to the total intensity and circular polarization are then

$$
C_{I}=C_{V}=\frac{1}{4} S_{\mathrm{N}, 0}\left[\left(S_{\mathrm{N}, 0}+4\left\langle I_{r_{o n}}\right\rangle\right)+\left(S_{\mathrm{N}, 0}+4\left\langle I_{l_{o n}}\right\rangle\right)\right]=\frac{1}{2} S_{\mathrm{N}, 0}\left(S_{\mathrm{N}, 0}+2 S_{\mathrm{S}, 0}\right) .
$$

\section{F.7. Derivation of Equation (48)}

Let the modulated instantaneous Stokes parameters $s^{\prime}=u s$ and the covariances between them

$$
\begin{aligned}
\mathbf{C}^{\prime} & =\left\langle s^{\prime} \otimes s^{\prime}\right\rangle-\left\langle s^{\prime}\right\rangle \otimes\left\langle s^{\prime}\right\rangle \\
& =\left\langle u^{2}\right\rangle\langle s \otimes s\rangle-\langle u\rangle^{2}\langle s\rangle \otimes\langle s\rangle
\end{aligned}
$$


where the latter equality arises because $s$ and $u$ are statistically independent (e.g. therefore, $\langle u s\rangle=\langle u\rangle\langle s\rangle$ ). Now let $u=\langle u\rangle+\delta u$ and $s=\langle s\rangle+\delta s$, which yields

$$
\begin{aligned}
\mathbf{C}^{\prime} & =\left(\langle u\rangle^{2}+\left\langle\delta u^{2}\right\rangle\right)(\langle s\rangle \otimes\langle s\rangle+\langle\delta s \otimes \delta s\rangle)-\langle u\rangle^{2}\langle s\rangle \otimes\langle s\rangle \\
& =\langle u\rangle^{2} \mathbf{C}+\varsigma_{u}^{2}\langle s\rangle \otimes\langle s\rangle+\varsigma_{u}^{2} \mathbf{C} \\
& =\left(\varsigma_{u}^{2}+1\right) \mathbf{C}+\varsigma_{u}^{2} S \otimes S
\end{aligned}
$$

where the first equality uses $\langle\delta u\rangle=0$ and $\langle\delta s\rangle=0$; the second equality uses $\left\langle\delta u^{2}\right\rangle=\varsigma_{u}^{2}$ and $\langle\delta s \otimes \delta s\rangle=\mathbf{C}$; and the last equality follows from $\langle u\rangle=1$ and $\langle s\rangle=S$.

\section{F.8. Derivation of Equation (49)}

After integration over $n^{\prime}$ instances of the instantaneous Stokes parameters, the sub-sample mean total intensity $\bar{S}_{0}^{\prime}$ has a population mean $S_{0} \equiv\left\langle\bar{S}_{0}^{\prime}\right\rangle=1$ and variance $\bar{\varsigma}_{0}^{\prime 2}=\left(2 n^{\prime}\right)^{-1}$. Each instance of the sub-sample mean is multiplied by a statistically independent and lognormally distributed variate $u$ with population mean $\langle u\rangle=\sqrt{\xi}$ and variance $\varsigma_{u}^{2}=\xi^{2}-\xi$, where $\xi=\exp \left(\varsigma^{2}\right)$ and $\varsigma$ is the standard deviation of the normally distributed variate $v$ used to generate $u=\exp (v)$.

The amplitude-modulated sub-sample mean total intensity $\bar{S}_{0}^{\prime \prime} \equiv u \bar{S}_{0}^{\prime}$ has population mean $\left\langle\bar{S}_{0}^{\prime \prime}\right\rangle=\langle u\rangle$, variance (Goodman 1960)

$$
\bar{\varsigma}_{0}^{\prime 2}=S_{0}^{2} \varsigma_{u}^{2}+\langle u\rangle^{2} \bar{\varsigma}_{0}^{2}+\varsigma_{u}^{2} \bar{\zeta}_{0}^{2}=\varsigma_{u}^{2}\left(1+\frac{1}{2 n^{\prime}}\right)+\langle u\rangle^{2} \frac{1}{2 n^{\prime}}
$$

and squared modulation index

$$
\beta^{\prime \prime 2} \equiv \frac{\bar{\varsigma}_{0}^{\prime \prime 2}}{\left\langle\bar{S}_{0}^{\prime \prime}\right\rangle^{2}}=\frac{\varsigma_{u}^{2}}{\langle u\rangle^{2}}\left(1+\frac{1}{2 n^{\prime}}\right)+\frac{1}{2 n^{\prime}} .
$$

After integrating over $N^{\prime}=n / n^{\prime}$ statistically independent instances of $\bar{S}_{0}^{\prime \prime}$, the square of the modulation index of the sample mean Stokes parameters $\bar{S}$ is given by

$$
\beta^{2}=\frac{\beta^{\prime \prime 2}}{N}=\frac{1}{n}\left[(\xi-1)\left(n^{\prime}+\frac{1}{2}\right)+\frac{1}{2}\right] .
$$

